\title{
EVOLUTION OF SPIRAL GALAXIES. VI. RADIAL DISTRIBUTIONS OF ABUNDANCES IN EXTERNAL GALAXIES
}

\author{
MerCedes Mollá \\ Departamento de Física Teórica, C-XI, Universidad Autónoma de Madrid, E-28049 Madrid, Spain;mercedes@astro1.ft.uam.es \\ FEDERICO FERRINI \\ Dipartimento di Fisica, Sezione di Astronomia e Astrofisica, Università di Pisa, Piazza Torricelli 2, I-56100 Pisa, Italy; \\ federico@astrpi.difi.unipi.it \\ AND \\ ANGELES I. DÍAZ \\ Departamento de Física Teórica, C-XI, Universidad Autónoma de Madrid, E-28049 Madrid, Spain; angeles@astro1.ft.uam.es \\ Received 1995 June 26; accepted 1996 February 15
}

\begin{abstract}
The multiphase model of Ferrini and coworkers has been used to study the evolution of six spiral galaxies of different morphological types. This kind of model has been successfully applied to describe the chemical evolution of the solar neighborhood, the whole Galactic disk, and the bulge of the Galaxy in the first five papers of this series. The present study constitutes an extension of our previous work to spiral galaxies other than our own, with a twofold goal: the investigation of the possible relation between the chemical evolution of galaxies and their classification, and the possibility of applying a unique evolutionary scheme to all galaxies. The comparison of results with the observed abundance gradients indicates that these are determined by a fundamental imprint that arises from an early phase of the evolution of a given galaxy: the initial mass of the protogalaxy and its spatial distribution. This suggests a connection between the present abundance gradient and the formation process of a spiral galaxy through the collapse of material forming the disk.
\end{abstract}

Subject headings: galaxies: abundances - galaxies: evolution - galaxies: spiral

\section{INTRODUCTION}

The existence of abundance radial gradients in spiral galaxies is a well-known observational fact (see, e.g., Pagel 1989a; Díaz 1989). However, the reasons for their existence are not well understood. Tinsley (1980) developed the basic system of equations that describes the chemical evolution of galaxies; the simple solution of considering the disk of a spiral galaxy as having been formed by a set of concentric regions that evolve like "closed boxes," although providing a certain amount of gradient through the radial variation of the gas fraction over the galactic disk, does not explain the observed gradients.

Different mechanisms can give rise to abundance radial gradients, and several of them could be simultaneously at work (Pagel 1989a; Götz \& Köppen 1992): (1) a radial variation of the yield, due either to a variation of the stellar productions (Peimbert \& Serrano 1982) or the initial mass function (IMF) (Güsten \& Mezger 1982); (2) a radial variation of the timescale for star formation; and (3) a radial variation of the timescale for infall of gas from outside the disk. Once an abundance gradient is established, it could be amplified or reduced by the presence of radial gas flows (Edmunds 1990; Götz \& Köppen 1992).

Models with infall of primordial gas are particularly appealing to reproduce the chemical evolution of our Galaxy: they are able to solve the " $\mathrm{G}$ dwarf" problem, they fit nicely into the classical scenario of collapse for the formation of galaxies, and they reproduce most of the data related to chemistry and kinematics, rotational velocity, and ages of stars.

Several numerical models that include infall can be found in the literature (Lacey \& Fall 1983, 1985; Clayton 1987, 1988; Wyse \& Silk 1989; Matteucci \& François 1989;
Sommer-Larsen \& Yoshii 1989; Pagel 1989b; Phillips \& Edmunds 1991). These models have been applied only to the Galactic disk, and yet they do not reproduce the observed gradients in a satisfactory way.

The gradients observed in external galaxies, as well as in the Milky Way, can be reproduced by models in which the ratio of the star formation rate (SFR) to infall rate (IR) varies with radius (Díaz \& Tosi 1984; Tosi \& Díaz 1985). These models do not yield real insight into the physical origin of gradients since both the SFR and the IR are represented by analytic functions without any clear physical meaning and without connection to a galactic evolution framework.

Modeling the chemical evolution of the Galaxy within a consistent scenario would require one to consider as first input the dynamical formation of the different structural components of the Galaxy (halo, bulge, and disk) as well as the formation of the radial structure of the disk. The key quantity determining the evolution is the SFR, and to provide a physical basis for the SFR, one must take into account the processes by which star formation takes place. These processes may have different probabilities of occurrence in different parts of the Galaxy and may also be different from galaxy to galaxy.

These requirements are the basis of the multiphase model developed by Ferrini, Shore, and collaborators (Ferrini et al. 1992, hereafter Paper I; Shore \& Ferrini 1995). Multipopulation, multizone models have been applied to the solar neighborhood, both without and with a thick disk (Pardi \& Ferrini 1994, hereafter Paper II; Pardi, Ferrini, \& Matteucci 1995, hereafter Paper IV), the whole Galactic disk (Ferrini et al. 1994, hereafter Paper III), and the Galactic bulge (Mollá \& Ferrini 1995, hereafter Paper V). 
In the present paper, we apply the same class of models to external galaxies of different Hubble types and luminosity classes. The same model that successfully explains the evolution of the Milky Way has been used. Section 2 describes the observational data for the sample of galaxies. In $\S 3$, the main model characteristics are summarized, together with the observational data used as inputs. The results are presented in $\S 4$ and discussed in $\S 5$. Finally, $\S 6$ summarizes the conclusions of this work.

In this paper we will not touch upon the question of heavy-metal distribution at times other than the present. This and the consequent time evolution will be addressed in a forthcoming paper.

\section{OBSERVATIONAL CONSTRAINTS}

Models of galactic evolution must calculate as large a number of quantities as possible in order to compare them with observational constraints. The nonlinear multipopulation model pertains to the category of models for galactic evolution that pretend, in order to reflect the intrinsic complexity of galactic structure, to fit all observational constraints, not only to yield a solution to selected problems. Now we analyze the observational data that may be compared with the model results.

The observational data related to the chemical evolution of spiral galaxies are (1) the radial distributions of gas (atomic and molecular), (2) the radial distributions of elemental abundances, (3) the radial distributions of the SFR, and (4) the time evolution of these quantities. These data are derived from observations in a variety of ways (e.g., emission-line spectral analysis, integrated photometry, radio observations). In particular, data about the SFR or the time evolution of elemental distribution are difficult to reconstruct reliably from observations. However, there are photometric analyses and metallicity determinations that constrain the possible histories of the SFR and the agemetallicity relation.

We have applied the multiphase model to six spiral galaxies. They were selected from the set of the nearest galaxies with a large number of data and with good spatial resolution. In order to compare the final results, we chose galaxies of different morphological types: NGC 224 (M31, type 3), NGC 628 (type 5), NGC 6946 (type 6), and NGC 300 (type 7). Together with the Milky Way (type 4), modeled in Paper III, they constitute a complete set. In order to compare galaxies with the same morphological type but with different luminosity classes, we have included two other galaxies, NGC 3198 (type 5) and NGC 598 (M33, type 6). They have the same types as NGC 618 and 6946, respectively, but their luminosity classes are different (see Table 1).

There are two other spiral galaxies for which there are a large number of data: NGC 5457 (M101) and NGC 2403. Both are of type 6, and they are very similar to NGC 6946 and 598, respectively, in abundances, size, luminosity, and, more importantly to us, in the radial distributions of these quantities. For this reason, they have been excluded from our sample. Our final list comprises the six galaxies for which we have collected data on the radial distributions of (1) atomic hydrogen, (2) molecular hydrogen, (3) elemental abundances, and (4) SFR.

1. Atomic hydrogen.-Radial distributions of atomic gas surface density, as derived from $21 \mathrm{~cm}$ radio observations, have been taken from the references given in Tables 2 and 3 .

2. Molecular hydrogen.-Molecular gas distributions are subject to large uncertainties in the method for their derivation and therefore deserve some comments. The molecular gas density is obtained from the $\mathrm{CO}$ line flux through a proportionality factor $\chi$, used to convert $\mathrm{CO}$ luminosities, $I(\mathrm{CO})$, to $\mathrm{H}_{2}$ column densities, $N\left(\mathrm{H}_{2}\right): \chi=N\left(\mathrm{H}_{2}\right) / I(\mathrm{CO})$. This factor has been estimated for the Milky Way by using different techniques--the measurement of the ${ }^{13} \mathrm{CO}$ emission, the correlation between the visual extinction in nearby dark clouds and the $\mathrm{CO}$ intensity, estimates of virial masses using ${ }^{12} \mathrm{CO}$ and ${ }^{13} \mathrm{CO}$ line widths and cloud sizes, and estimates of the total column density of nucleons derived from $\gamma$-ray observations. A detailed analysis (Bronfman et al. 1988; Sanders, Solomon, \& Scoville 1984, and references therein) of the conversion factors found by some authors shows that all of them are in a range between $1.8 \times 10^{20}$ and $7.8 \times 10^{20} \mathrm{~cm}^{-2}\left(\mathrm{~K} \mathrm{~km} \mathrm{~s}^{-1}\right)^{-1}$. Hence, data from different authors obtained with different values of $\chi$ produce dissimilar distributions.

In the case of our Galaxy, it seems that this factor has a radial dependence along the disk (Mead, Kutner, \& Evans 1990; Sodroski 1991). Tosi \& Díaz (1985) analyzed the effects of using molecular surface density distributions obtained with $\chi$ both constant and varying with radius, and they showed that a radial dependence of $\chi$, because of the radial variation of the metallicity, is needed in order to reproduce the observed gas fraction radial distribution. In particular, the larger abundance of carbon in the inner parts of galaxies may increase the probability of collisions between $\mathrm{H}_{2}$ and $\mathrm{CO}$ molecules, producing a larger $\mathrm{CO}$ intensity. $\mathrm{H}_{2}$ masses derived by using a constant pro-

TABLE 1

ObSERVATIONAL ChaRaCteristics

\begin{tabular}{|c|c|c|c|c|c|c|c|c|c|c|c|c|}
\hline \multirow[b]{2}{*}{ GALAXY } & \multicolumn{4}{|c|}{ TYPE } & \multirow[b]{2}{*}{$\begin{array}{c}\text { DisTaNCE } \\
(\mathrm{Mpc})\end{array}$} & \multirow[b]{2}{*}{$\begin{array}{c}R_{\text {eff }} \\
(\mathrm{kpc})\end{array}$} & \multirow[b]{2}{*}{$\begin{array}{c}R_{s} \\
(\mathrm{kpc})\end{array}$} & \multirow[b]{2}{*}{$\begin{array}{c}R_{B} \\
(\mathrm{kpc})\end{array}$} & \multirow[b]{2}{*}{$\begin{array}{c}R_{\text {iso }} \\
(\mathrm{kpc})\end{array}$} & \multirow[b]{2}{*}{$\begin{array}{c}V_{\text {rot }} \\
\left(\mathrm{km} \mathrm{s}^{-1}\right)\end{array}$} & \multirow[b]{2}{*}{$\log M_{\text {tot }}$} & \multirow[b]{2}{*}{$M_{\mathrm{H}} / M_{\text {tot }}$} \\
\hline & (1) & (2) & (3) & (4) & & & & & & & & \\
\hline NGC 224 (M31)...... & 3 & $\mathrm{Sb} \mathbf{I}-\mathrm{II}$ & $\cdots$ & $\mathrm{Sb}$ & 0.69 & 7.81 & 5.38 & 5 & 31 & 250 & 11.37 & 0.018 \\
\hline NGC $300 \ldots \ldots \ldots \ldots$ & 7 & Sc(s) II & 5 & $\mathrm{Scd} / \mathrm{Sd}$ & 1.65 & 2.38 & 1.70 & 0.0 & 9.7 & 80 & 9.89 & 0.102 \\
\hline NGC 598 (M33)...... & 6 & Sc(s) II-III & 5 & $\mathrm{Scd} / \mathrm{Sc}$ & 0.72 & 2.55 & 1.72 & 0.5 & 11.8 & 85 & 10.10 & 0.099 \\
\hline NGC $628 \ldots \ldots \ldots \ldots$ & 5 & $\mathrm{Sc}(\mathrm{s}) \mathrm{I}-\mathrm{II}$ & 9 & $\mathrm{Sc}$ & 7.2 & 5.03 & 2.67 & 2 & 14.66 & 220 & 11.35 & 0.035 \\
\hline NGC $3198 \ldots \ldots \ldots \ldots$ & 5 & $\mathrm{Sc}(\mathrm{s}) \mathrm{I}-\mathrm{II}$ & $\ldots$ & Sc & 9.6 & 4.50 & 2.68 & 1.5 & 24.57 & 160 & 10.77 & 0.095 \\
\hline NGC $6946 \ldots \ldots \ldots \ldots$ & 6 & $\mathrm{Sc}(\mathrm{s}) \mathrm{I}-\mathrm{II}$ & 9 & Scd & 5.7 & 5.09 & 4.54 & 0.5 & 18.57 & 180 & 10.81 & 0.097 \\
\hline
\end{tabular}

NoTES.-Observational characteristics for the six selected spiral galaxies (Tully 1988; Simien \& de Vaucouleurs 1986; Huchtmeier \& Ritcher 1989). The types are according to (1) Simien \& de Vaucouleurs, (2) Sandage, (3) arm class according to Elmegreen \& Elmegreen 1987, and (4) Hubble. The quantities in the remaining columns are, respectively, the disk photometric effective radius, $\boldsymbol{R}_{\text {eff }}$ (Vila-Costas $\&$ Edmunds 1992); disk scale length of the surface brightness, $R_{s}$ (Zaritsky et al. 1994, except for NGC 598, from Minniti, Olszewski, \& Rieke 1994); bulge radius, $R_{B}$ (Simien \& de Vaucouleurs 1986); isophotal radius, $R_{\text {iso }}$ (Tully 1988); maximum rotational velocity, $V_{\text {rot }}$ (from references in Table 3); estimated total mass, $M_{\text {tot }}$, in $M_{\odot}$; and observed ratio of atomic gas to total mass, $M_{\mathrm{H}} / M_{\text {tot }}$, both from Tully 1988 . 
TABLE 2

References to Observational Data: Key to AbBreviations

\begin{tabular}{|c|c|c|c|}
\hline Abbreviation & Reference & Objects & Data \\
\hline AL. & Adler \& Liszt 1989 & $\mathrm{CO}$ & Gas distributions \\
\hline BG. & Begeman 1987 & $\mathrm{H}_{\mathbf{I}}$ & Rotation curve \\
\hline BL . & Belley \& Roy 1992 & H II regions & Abundances \\
\hline BN. & Bonnarel et al. 1988 & Gas & Rotation curve \\
\hline BR . & Braun 1991 & Gas & Rotation curve \\
\hline & Carignan \& Freeman 1985 & Gas & Rotation curve \\
\hline CO. & Corradi et al. 1991 & $\mathrm{H} \alpha$ flux & SFR \\
\hline DB. & Dennefeld \& Kunth 1981; Blair et al. 1981 & $\mathrm{H}$ II regions & Abundances \\
\hline DG & DeGioia-Eastwood et al. 1984 & $\mathrm{H} \alpha$ flux & SFR \\
\hline DH & Deharveng et al. 1988 & H II regions & Abundances, SFR \\
\hline KA. & Kwitter \& Aller 1981 & $\mathrm{H}$ II regions & Abundances \\
\hline $\mathbf{K B}$. & Kamphuis \& Briggs 1992 & Gas & Rotation curve \\
\hline KN & Kennicutt 1989 & $H \alpha$ flux & SFR \\
\hline KO & Koper et al. 1991 & $\mathrm{H} \mathrm{I,} \mathrm{CO}$ & Gas distributions \\
\hline MC & McCall et al. 1985 & H II regions & Abundances \\
\hline NT . & Natali et al. 1992 & $B-V, U-B$ & SFR \\
\hline NW. & Newton 1980 & $\mathbf{H}_{\mathbf{I}}$ & Gas distributions \\
\hline PG. & Pagel et al. 1979 & H II regions & Abundances \\
\hline RG. & Rogstad et al. 1979 & H I & Gas distributions \\
\hline RK. & Rumstay \& Kaufman 1983 & Solar neighborhood counts & SFR \\
\hline SG . & Sage 1993 & $\mathrm{CO}$ & Gas distributions \\
\hline TY . & Tacconi \& Young 1986 & $\mathrm{H} \mathrm{I}, \mathrm{CO}$ & Gas distributions \\
\hline VI... & Vílchez et al. 1988 & H II regions & Abundances \\
\hline WB & Walterbos \& Braun 1992 & $\mathrm{H} \alpha$ flux & SFR \\
\hline WE . & Wevers et al. 1986 & $\mathrm{HI}_{\mathrm{I}}$ & Gas distributions \\
\hline YO. & Young \& Scoville 1982 & $\mathrm{CO}$ & Gas distributions \\
\hline ZA .. & Zaritsky et al. 1994 & H II regions & Abundances \\
\hline ZR . & Zaritsky, Elston, \& Hill 1989 & Gas & Rotation curve \\
\hline
\end{tabular}

portionality factor would then be overestimated in the central regions of galaxies. This effect can be accounted for by assuming a radial dependence for $\chi$ of the form

$$
\chi(R)=\left.\frac{N\left(\mathrm{H}_{2}\right)}{I(\mathrm{CO})}\right|_{R}=\chi_{0} \frac{\mathrm{O} / \mathrm{H}\left(R_{0}\right)}{\mathrm{O} / \mathrm{H}(R)} .
$$

For our Galaxy, the subscript zero refers to quantities evaluated at the solar Galactocentric radius $\boldsymbol{R}_{\mathbf{0}}$. For external galaxies, it represents the "equivalent solar radius" (see $\S 3.2$. This value may be different from galaxy to galaxy. NGC 628 and 6946 are similar to our Galaxy in metallicity and size, and therefore the same value of $\chi_{0}$ and its radial dependence have been assumed (Paper III), modifying their molecular hydrogen distributions accordingly. For the other two galaxies with data, the $\chi_{0}$-factor is unknown. The original distribution by Koper et al. (1991) can be used for NGC 224 since the observed metallicity distribution for this galaxy is rather flat (see $\S 4$ ).

NGC 598 shows, however, a steeper oxygen radial variation. Therefore, using a factor $\chi(R)$ that depends on the metallicity as in equation (1) would modify the molecular gas surface density $\sigma_{\mathrm{H}_{2}}(R)$, decreasing the central value and increasing the values for $R \geq 1 \mathrm{kpc}$, thus transforming the original exponential form into a flatter distribution. It should be noted that no data exist for this galaxy for $R \geq 1$ kpc. Data exist (Young \& Scoville 1982), but only for the central region. With these values used for normalization purposes, and assuming a radial distribution that follows the blue luminosity (in agreement with results from Young, Scoville, \& Brady 1985), Tosi \& Díaz (1985) estimated a radial distribution of the molecular gas surface density. Recently, Deul \& den Hartog (1990), studying the H I holes of the disk in this galaxy, estimated a molecular gas mass at least a factor of 3 larger than that obtained using the observed CO intensity and a solar $\chi$-factor. This implies a lower number of $\mathrm{CO}-\mathrm{H}_{2}$ interactions, as a result of a lower carbon abundance, in agreement with the argument above. Therefore, to obtain the molecular radial distribution, we have taken Tosi \& Díaz's, but multiplied by a factor of 3 for $R \geq 1 \mathrm{kpc}$.

Recently, other authors have obtained data along this same line: Verter \& Hodge (1995) and Wilson (1995) estimated the molecular mass in nearby low-metallicity gal-

TABLE 3

ReFERENCES TO DATA FOR INPUT OR FOR COMPARISON WITH MODELS

\begin{tabular}{ccccc}
\hline \hline Galaxy & Rotation Curves & Gas Distribution & Abundances & SF Surface Density \\
\hline NGC 224 $\ldots \ldots .$. & BR & KO & DB & WB \\
NGC 300..... & CF & RG & PG, DH & DH \\
NGC 598 $\ldots \ldots$. & ZR & NW, YO & VI, KA & KN, RK \\
NGC 628 $\ldots \ldots$. & KB & WE, SG, AL & MC, BL & NT \\
NGC 3198..... & BG & WE & ZA & CO \\
NGC 6946..... & BN & TY & MC, BL & KN, DG \\
\hline
\end{tabular}


axies, where it is possible to resolve individual clouds, and compared their virial masses with the observed $\mathrm{CO}$ luminosities. In this way, they obtained a CO-to- $\mathrm{H}_{2}$ conversion factor $(\chi)$ that changes with metallicity. For low metallicities, this factor increases. For GR 8, Verter \& Hodge estimated a factor of 20 increase for an oxygen abundance $3 \%$ of solar. In particular, Wilson showed a calibration in which $\log \left(\chi / \chi_{0}\right)$ decreases linearly with increasing oxygen abundance $[12+\log (\mathrm{O} / \mathrm{H})]$ with a slope of -0.67 . If we apply this new calibration to the published data of molecular masses and take into account the oxygen abundance radial distributions (see next paragraph), we obtain $\mathrm{H}_{2}$ radial distributions very similar to those calculated with Tosi $\&$ Díaz's method, which we hence adopt.

3. Elemental abundances.-The abundance radial distributions at present have been taken from the references given in Table 2. They were derived from observations of $\mathrm{H}$ II region emission lines. For most $\mathrm{H}$ iI regions the data refer to oxygen, but in some cases abundances for other elements (mainly nitrogen, sulphur, or both) are given.

There are well-determined metallicities for a good number of objects, but, because of selection effects and the method of analysis used, these correspond to the brightest and highest excitation objects, which allow the detection and measurement of the weak auroral lines needed for the determination of the electron temperature and hence of the metal abundances. Most of the $\mathrm{H}$ in regions that fall into this category lie in the outer parts of the galactic disks.

An empirical calibration of the parameter $R_{23} \equiv$ $([\mathrm{O} \mathrm{II}]+[\mathrm{O} \mathrm{III}]) / \mathrm{H} \beta$ (Pagel et al. 1979) with oxygen abundance has allowed the determination of metallicities up to the solar value. This is the range of metallicities found in most inner $\mathrm{H}$ II regions. Unfortunately, the calibration is not valid for higher metallicities since the basic mechanism at work, i.e., the fact that the optical [O II] and [O III] lines act as the main coolants for the nebula, is no longer true. In fact, at metallicities higher than solar, the infrared lines become increasingly important, and other elements besides oxygen start to contribute significantly to the cooling. The three-parameter behavior of $\mathbf{H}$ II regions now becomes evident, and the determination of the metallicity requires in addition the simultaneous determination of the ionization parameter and the temperature of the ionizing source (Vílchez \& Pagel 1988; Díaz et al. 1991; Díaz 1994). Therefore the empirical method cannot be used to determine metallicities higher than solar, and published oversolar abundances derived with this method cannot be considered to be established (see Oey \& Kennicutt 1993).

These difficulties in the determination of abundances introduces a bias in the derivation of the radial distribution of abundances toward the outer part of the disk, with the inner region being severely undersampled.

Keeping this caveat in mind, in general, existing data show a dispersion larger than expected from measurement uncertainties. This point has been largely analyzed for the oxygen abundances of $\mathbf{H}$ II regions in the Milky Way (Lambert 1989; Meyer 1989) without a clear conclusion, even when a large set of data exists and comparisons with stellar metallicity data can be made. A possible explanation relates to the depletion of oxygen from the gas phase, it being trapped in solid grains. If this is the case, the interstellar abundances would be underestimated.

4. SFR.-For the present-day radial distribution of the SFR, we have estimates for all the sample galaxies. For
NGC 628 and 6946, published radial distributions of the SFR surface density exist (Table 3). For the other galaxies, NGC 224, 300, 598, and 3198, they have been derived from published $\mathrm{H} \alpha$ emission fluxes from Walterbos \& Braun (1992), Deharveng et al. (1988), Kennicutt (1989), and Corradi et al. (1991), respectively, using the method described by Wilson \& Scoville (1991).

\section{EVOLUTION MODEL}

The evolution model has already been described in detail in Papers I and III. The initial condition is a spheroid of primordial gas. The model for every galaxy in our sample is similar to that employed for the Milky Way: a sphere defined by the total radius of the galaxy. We assume that the primordial gas of the spheroid collapses onto the galactic plane and forms the disk. This sphere is divided into concentric cylindrical regions $1 \mathrm{kpc}$ wide, with a height determined by the corresponding galactocentric distance on the disk and the total radius of the sphere.

As the total radius for each galaxy, we adopt the value 30 $\mathrm{kpc}$, although calculations have been performed up to the isophotal radii (Tully 1988), since no data are present for our purposes outside it. Other useful observational properties of the six selected galaxies, taken from the Sandage \& Tammann (1987) and Huchtmeier \& Ritcher (1989) catalogs and from Elmegreen \& Elmegreen (1987), Vila-Costas \& Edmunds (1992), and Zaritsky, Kennicutt, \& Huchra (1994), are summarized in Table 1.

In the direction perpendicular to the disk, the model has two zones, and into each of them we allow for different phases of matter aggregation: diffuse gas $(g)$, clouds $(c)$, lowmass stars $\left(s_{1} ; m<4 M_{\odot}\right)$ and high-mass stars $\left(s_{2}\right)$, and remnants $(r)$. The mass in the different phases changes through several conversion processes, defined and discussed in detail in previous papers of this series, namely:

Cloud formation from diffuse gas $\left(\propto g^{n}, n \simeq 1.5\right)$;

Star formation from cloud collisions $\left(\propto c^{2}\right)$;

Diffuse gas restitution from cloud collisions $\left(\propto c^{2}\right)$;

Induced star formation via massive star-cloud interactions $\left(\propto c s_{2}\right)$;

Diffuse gas restitution from induced star formation $\left(\propto c s_{2}\right)$;

Accumulation of diffuse gas in the disk from the corresponding halo region $\left(\propto g_{H}\right)$;

Restitution of enriched material from dying stars, considering their nucleosynthesis, their initial mass function (and the delayed restitution), and their final fate, via a "quiet" evolution or Type I or II supernova explosions $\left(D_{1}\right.$ and $D_{2}$ are the low-mass and high-mass stars' death rates, respectively, while $W_{j}$ is the restitution of element $j$ and $W$ is the total restitution to the diffuse gas phase).

The complete set of equations for each zone is (the subscript $i$ refers to halo or disk, and, for simplicity, we do not introduce the index corresponding to different radial positions):

$$
\begin{gathered}
\frac{d s_{1, i}}{d t}=H_{1, i} c_{i}^{2}+a_{1, i} c_{i} s_{2, i}-D_{1, i}, \\
\frac{d s_{2, i}}{d t}=H_{2, i} c_{i}^{2}+a_{2, i} c_{i} s_{2, i}-D_{2, i}, \\
\frac{d g_{i}}{d t}=-\mu_{i} g_{i}^{n}+a_{i}^{\prime} c_{i} s_{2, i}+H_{i}^{\prime} c_{i}^{2}+W_{i}+\mathscr{F}_{i},
\end{gathered}
$$


$\frac{d c_{i}}{d t}=\mu_{i} g_{i}^{n}-\left(a_{1, i}+a_{2, i}+a_{i}^{\prime}\right) c_{i} s_{2, i}-\left(H_{1, i}+H_{2, i}+H_{i}^{\prime}\right) c_{i}^{2}$

$$
\frac{d r_{i}}{d t}=D_{1, i}+D_{2, i}-W_{i},
$$

where $\mu_{i}$ is the coefficient of cloud formation from diffuse gas, $H_{i}$ is the coefficient for star formation by cloud collisions, and $a_{i}$ is the coefficient for induced star formation.

The function $\mathscr{F}_{i}$ is a loss term of $-f g_{H}$ if describing the halo; it appears in the evolution equation for the disk gas as a gain term with the opposite sign. The equations for the elemental abundances $X_{j}$ are written according to the standard approach (see, e.g., Tinsley 1980), from

$$
\frac{d\left[X_{j, i}\left(g_{i}+c_{i}\right)\right]}{d t}=-\psi_{i} X_{j, i}+\mathscr{F}_{i} X_{j, i}+W_{j, i},
$$

where now the star formation rate $\psi_{i}$ is determined selfconsistently in each zone by the evolution of phases as

$$
\psi_{i}=\left(H_{1, i}+H_{2, i}\right) c_{i}^{2}+\left(a_{1, i}+a_{2, i}\right) c_{i} s_{2, i} .
$$

We do not introduce the thick disk zone, the intermediate population between the halo and the disk in our Galaxy, because it is not clear whether in the galaxies of our sample a thick disk zone exists.

In principle, it is possible to introduce radial flows, diffusion terms, and other interactions within different zones (Shore 1983; Shore \& Ferrini 1995), but since our aim is to investigate the simplest mechanisms of the formation of abundance gradients, we do not at present introduce these terms in the formalism.

From the point of view of the multiphase model, the main differences among galaxies will reside in (1) the initial conditions of the mass distribution and large-scale dynamical aspects, which would influence the coupling between the zones (via the term $\mathscr{F}$ ), and (2) the values of the coupling constants that appear in the system equations, which would be a function of the "microphysics" involved in the star formation processes. This argument implies that we must fix, guided by observations and theoretical studies, the values of the parameters $H, a, \mu$, and $f$ for each galaxy (about this parameter selection and the problem of the uniqueness of the model, see the Appendix) and as functions of the position inside each galaxy.

\subsection{Mass Distributions in the Sample Galaxies}

We assume for simplicity that there have been no mass exchanges with the intergalactic medium or between radial zones, and let our galaxies evolve as closed systems. The simplest way to estimate the mass distribution inside spiral galaxies that satisfies the observed rotation curves is to use the expression $M(R) \simeq R V^{2}(R) / G$ (Lequeux 1983), where $R$ is the radius, $M(R)$ is the mass within a given radius $R$, and $V(R)$ is the rotational velocity. This approach is valid for reasonable geometries (Campos-Aguilar, Prieto, \& Garcia 1993).

References for the rotation curves used for each galaxy are given in Table 3. For NGC 628 and 6946, the rotation curves had been obtained for distances of 10.4 and 10.1 $\mathrm{Mpc}$, respectively. We have scaled these distributions to the more recent estimates of 7.2 and $5.7 \mathrm{Mpc}$ used for the abundance and gas distribution data referred to in $\S 2$.
The mass quoted for each galactocentric radius is the present total mass, not just the disk mass. It has a spheroidal component and a disk component, as opposed to the classical disk distribution usually used in chemical evolution models for the galactic disk.

Only visible matter takes part in the chemical evolution. Dark matter influences the coupling between the halo and the disk, with its dynamical influence on the timescale for accumulation of gas onto the disk (calculated in $\S 3.2$ ), which would be reduced. This has an effect on the rate of star formation in the disk and on the rate of elemental production, but it is clearly a second-order correction to the scheme and is part of the uncertainties in the only true free parameter in the multiphase model, $f$. Dark matter is dominant in the outer parts of galaxies, while the model is applied to the luminous disk, within the isophotal radius, where dark and baryonic components are approximately equivalent. In other words, our initial time for the formation of the disk is the time at which the baryons have already collapsed from the protogalactic structure to the region corresponding to the present baryonic halo. Assuming the conspiracy between dark and baryonic mass distributions, we consider that there is a constant ratio between the two within the isophotal radius, corresponding to a scaling of the parameter $f$. Ferrini \& Poggianti (1993) discussed instead the importance of dark matter for models in which outflows are allowed: the presence of dark matter on larger scales is fundamental to the fate of the expelled gas.

\subsection{Variation of the Disk Accumulation from the Halo}

The halo gas collapses onto the disk at a rate that depends on the inverse of the free-fall time, which, in turn, depends on the galactocentric distance. The gas is accreted more rapidly in the central regions than in the external parts of the disk. This radial dependence results naturally when one considers that the collapse timescale for a given mass to form a flat disk is $\tau_{\mathrm{ff}} \propto \sigma^{-1 / 2}$ (Jones \& Wyse 1983), where $\sigma$ is the mass surface density. For an exponential disk, this yields a behavior for the collapse time that is an exponentially increasing function of the galactocentric radius $R$, $\tau(R)=\tau_{0} e^{R / l}$-in agreement with other authors' assumptions (Lacey \& Fall 1985; Götz \& Köppen 1992)-with a scale length equal to one-half the scale length for the surface density distribution. Taking into account that the observed mass surface density scale length is $4 \mathrm{kpc}$ for the Milky Way, we obtain $l=2 \mathrm{kpc}$. This value has been used for all galaxies. In Paper III, we discussed the effect of changing $l$ by a factor of 4 , concluding that the increase does not produce large differences in the results. In the following we hence consider, for simplicity, that the entire dependence on galactic mass is contained in the multiplicative factor. In order to check the effect of this hypothesis, we have computed the final surface density of the disk and compared it with the observed one in each galaxy, finding good agreement.

The value of $\tau_{0}$ for each galaxy was determined from the value obtained for the solar neighborhood (Paper II), $\tau_{\odot}=$ $4 \mathrm{Gyr}$, assuming a relation between the free-fall time and the total mass of the form $\tau_{0} \propto M_{9}^{-1 / 2} T$ (Gallagher, Hunter, \& Tutukov 1984), where $M_{9}$ is the total mass of the galaxy in $10^{9} M_{\odot}$ and $T$ is its age, assumed to be $13 \mathrm{Gyr}$. Therefore

$$
\tau_{0}=\tau_{\odot}\left(M_{9, \mathrm{gal}} / M_{9, \mathrm{MWG}}\right)^{-1 / 2},
$$


where the subscript MWG refers to the Milky Way, providing a value for the collapse-time characteristic for every selected galaxy that is associated with a region equivalent to the solar neighborhood in our Galaxy. This region is located at a galactocentric distance $R_{0}$, which is calculated from the photometric radii by using the relation $R_{\odot} / R_{0}=$ $R_{\text {eff, MwG }} / R_{\text {eff,gal }}$. The values of $R_{0}$ for the sample galaxies are listed in Table 4.

\subsection{Variations of the Star and Cloud Formation Coupling Coefficients}

We discussed in Paper III the laws of variations of the coefficients $H, a$, and $\mu$ with galactocentric radius in our Galaxy. These can be considered to be the product of the typical timescale of the corresponding physical process and the efficiency of the same process ( $\epsilon$; see Paper I). The timescales can be calculated explicitly with simple theoretical arguments and evaluated from the numerous observations on the processes in the solar neighborhood; they are naturally functions of Galactocentric distance. Under the hypothesis that the same physics rules the solar vicinities as well as every region inside galaxies, we assume that the analytical expressions derived in Paper III will be valid in all the galaxies of our sample. Hence, we summarize:

1. The coefficient of cloud formation from diffuse gas is $\mu_{i} \propto \epsilon_{\mu, i}\left[V_{i}(R)\right]^{-1 / 2}$, where $V_{i}(R)$ is the volume of the halo or disk at each radial ring and $\epsilon_{\mu, i}$ is the efficiency of the process of formation of molecular clouds from diffuse gas, in each ring.

2. The coefficient for star formation by cloud collisions is $H_{i} \propto \epsilon_{H, i}\left[V_{i}(R)\right]^{-1}$, where $\epsilon_{H, i}$ is the efficiency of forming stars by cloud collisions (explicitly, the ratio of mass in stars to the initial mass of clouds).

3. The coefficient for induced star formation is $a_{i} \propto \epsilon_{a, i}$, where $\epsilon_{a, i}$ is the efficiency of induced star formation. It describes a local process, due to the interactions of molecular clouds with nearby massive stars, so it can be considered to be independent of position.

The specification of the geometrical data for every galaxy determines the coefficients as a function of position since they depend only on the volume.

The efficiencies that appear in the previous relations must be a function of the different morphological types of galaxies, if these reflect differences in their main physical properties (see Sandage 1986). We assume their variation with morphological type, as established by Ferrini \& Galli (1988), according to whom gravitation through the spiral density wave (SDW) changes the conditions of star formation in each subgalactic region (our rings); the main actions of the SDW are that
The spiral arm shock can compress the diffuse gas phase, favoring the formation of clouds; and

The spiral perturbation behaves like a potential well, focusing clouds, changing their velocity distribution, and increasing the frequency of cloud-cloud collisions.

In summary, a stronger SDW will increase the efficiency of cloud formation and cloud-cloud collisions and hence will increase $\epsilon_{\mu}$ and $\epsilon_{H}$. To quantify these variations, Ferrini \& Galli (1988) referred to particle simulations of a clumpy interstellar medium, suggesting a variation in the efficiencies by a factor of 10 from one Hubble type to the other, with the highest values for Sa types. The calibration was obviously performed on the Milky Way.

The percolation models, based on induced star formation, described in our scheme by the term $a c s_{2}$, suggest instead that the typical efficiencies of this local process, which induce organization of large-scale structures, must be constant; hence we consider $\epsilon_{a}$ to be constant for every zone and galaxy.

To update and confirm the Ferrini \& Galli (1988) analysis, we took advantage of detailed studies of starforming regions. Lada et al. (1988) analyzed the conversion of atomic gas to molecular clouds in a region of M31 at 7 $\mathrm{kpc}$ from the galaxy's center, obtaining an efficiency of $50 \%-70 \%$. This implies a value of $\epsilon_{\mu}=0.50-0.70$, very similar to the value $\epsilon_{\mu}=0.45-0.70$ resulting from the Ferrini \& Galli scheme, adopting an efficiency $\epsilon_{\mu}=0.08$ for the Galaxy and an increasing factor of 5-8 from $\mathrm{Sbc}$ to $\mathrm{Sb}$ galaxies.

The other galaxy with a number of studies of starforming regions is NGC 598 (M33). For this galaxy, Wilson et al. (1988), Wilson \& Scoville (1991), and Wilson, Scoville, \& Rice (1991) estimated a mean time for the consumption of molecular gas of $\sim 1.1 \mathrm{Gyr}$ and a mean time for the consumption of the total gas of $\sim 1.4 \mathrm{Gyr}$ (Young et al. 1986 found a time of consumption of gas of $5.6 \mathrm{Gyr}$ ). This implies $\epsilon_{\mu}=0.04-0.05$ and $\epsilon_{H^{\prime}}=0.01$. The corresponding values found using Ferrini \& Galli's method are $\epsilon_{\mu}=0.04$ and $\epsilon_{H^{\prime}}=0.005-0.01$, with decreasing factors of 2 and 5, respectively, from $\mathrm{Sbc}$ to $\mathrm{Scd}$ galaxy values.

There are also estimates of the star formation efficiency, defined to be the SFR per hydrogen mass unit, from Tacconi \& Young (1989) for NGC 6946. Following these authors, a radial dependence of the cloud formation efficiency $\left(\epsilon_{\mu}\right)$ exists, with it being a factor of 10 larger in the inner regions of this galaxy than in the outer ones, while the formation of massive stars has an efficiency $\left(\epsilon_{H}\right)$ that is almost constant along the disk. This same kind of behavior is assumed in the present work, with a radial dependence of the efficiency $\epsilon_{\mu}$ yielding larger values at inner radii and a

TABLE 4

MODel PARAMETERS

\begin{tabular}{|c|c|c|c|c|c|c|c|}
\hline Galaxy & Type & $\begin{array}{c}V_{\text {rot,max }} \\
\left(\mathrm{km} \mathrm{s}^{-1}\right)\end{array}$ & $\begin{array}{c}R_{0} \\
(\mathrm{kpc})\end{array}$ & $\begin{array}{c}M_{\text {tot }}\left(R_{0}\right) \\
\left(10^{9} M_{\odot}\right)\end{array}$ & $\begin{array}{c}\tau_{\text {coll }}\left(R_{0}\right) \\
(\mathrm{Gyr})\end{array}$ & $\epsilon_{\mu}$ & $\epsilon_{H^{\prime}}$ \\
\hline Milky Way...... & 4 & 220 & 8 & 200 & 4 & 0.08 & 0.15 \\
\hline NGC $224 \ldots \ldots \ldots$ & 3 & 250 & 10 & 235 & 3 & 0.45 & 0.50 \\
\hline NGC $300 \ldots \ldots \ldots$ & 7 & 80 & 2 & 10 & 15 & 0.07 & 0.007 \\
\hline NGC $598 \ldots \ldots \ldots$ & 6 & 85 & 3 & 13 & 8 & 0.05 & 0.005 \\
\hline NGC $628 \ldots . . .$. & 5 & 220 & 6 & 220 & 4 & 0.25 & 0.01 \\
\hline NGC $3198 \ldots . .$. & 5 & 150 & 4 & 75 & 5 & 0.20 & 0.02 \\
\hline NGC $6946 \ldots \ldots$. & 6 & 180 & 5 & 74 & 6 & 0.18 & 0.02 \\
\hline
\end{tabular}

Notes. $-R_{0}$ is the "equivalent" solar radius; $M_{\text {tot }}\left(R_{0}\right)$ and $\tau_{\text {coll }}\left(R_{0}\right)$ are the corresponding galactic mass and collapse timescale. 
constant $\epsilon_{H}$. In Table 4, we show the values finally adopted for the model efficiencies.

\section{RESULTS}

The model, with the prescriptions for the coupling constants and the initial conditions for the mass distribution, can follow in each zone the evolution of the distinct phases and the fractional abundances of 15 elements. Having sliced the galaxies into concentric cylindrical regions $1 \mathrm{kpc}$ wide in the galactic plane, we also obtain radial distributions for mass densities in every phase and for elemental abundances.

We may consider that the gas fractions, both in the diffuse and in the cloud phases, are the most sensitive internal clocks of the evolution of galaxies as far as these galaxies may be considered to be isolated. Indeed, the gas-phase evolution reflects the entire dynamics in play: the formation of the disk, the consumption of gas by the star formation, and the restitution of gas from stars. Since part of the mass will be frozen into remnants, whose fraction can only increase during galactic evolution, the total gas mass will asymptotically decrease. However, it is not the case that both phases of gas (atomic or diffuse and molecular or cloudy) will always have a negative derivative with respect to time: the two phases will exchange mass at rates that depend not only on star formation but also on the typical timescales for cloud-forming instabilities and on the collapse timescale from the halo; this mass exchange is a major difference of our model from non-self-regulating models, which would be unable to describe this complex history.

Standard models force the evolution by choosing, a priori, the star formation history and result generally in a temporally exponential decrease of gas; this produces uncomfortably short-lived galaxies and the inability to reproduce typical observational constraints in the solar neighborhood, such as the G-dwarf metallicity distribution, without ad hoc assumptions. Furthermore, standard models usually do not take into account the cloud phase; it is obvious that no clouds means no stars, and this is a formal omission that probably reduces the reliability of those models. The comparison of model results with observed gas distributions can then help to fix the age of the galactic component with which these distributions are associated. To use the gas evolution timescale rather than the heavy-metal evolution is preferred, because two independent ingredients influence the elemental growth rate: the stellar lifetimes and nucleosynthesis, and the efficiency of mixing. We will not discuss these questions in the present paper, adopting instead a rather standard set of hypotheses, described in the previous papers of the series.

In what follows, we present the results for each galaxy separately, while in $\S 5$ we discuss how these results fit into the general correlations found by different authors.

\subsection{NGC 224}

In Figures $1 a$ and $1 b$, we plot the time evolution of surface densities of $g$ and $c$ for five different times. The comparison of the observed and theoretical distributions in Figure 1 suggests that the age of the disk of NGC 224 is $\sim 13$ Gyr. Model results for neutral hydrogen fit the observed distribution perfectly (Koper et al. 1991). For molecular hydrogen (Fig. 1b), the shape of the distribution is well reproduced, the calculated absolute densities are of the same order of magnitude as the observed ones within a factor of 2, and, taking into account the uncertainties in the
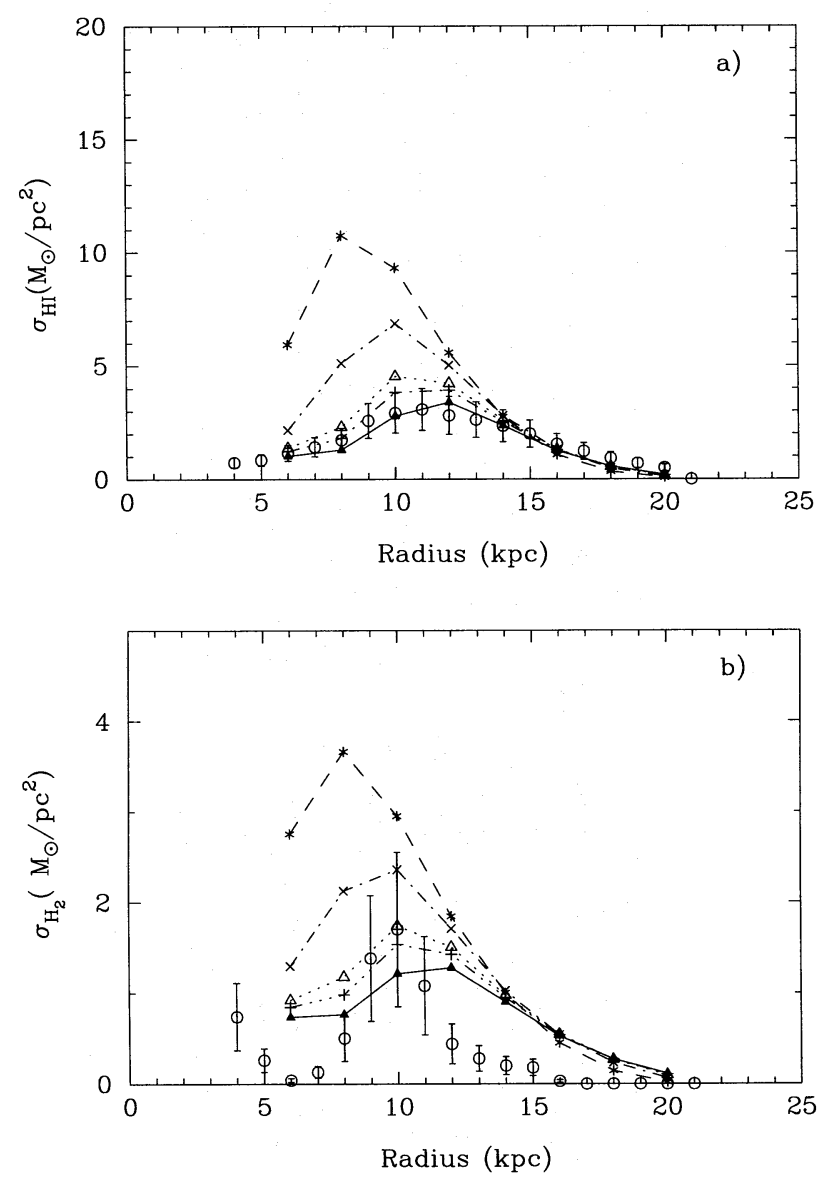

FIG. 1.-Radial distributions of gas surface densities for NGC 224: (a) diffuse gas or atomic component and (b) molecular clouds, at five times2.5 (asterisks), 5 (crosses), 8.5 (open triangles), 10 (plus signs), and $13 \mathrm{Gyr}$ (filled triangles) - and observational data from Koper et al. (1991; circles).

molecular gas density estimates, these results may be considered to be good enough.

The radial distribution of the oxygen abundance evaluated by our model follows the observed distribution rather well (Fig. 2a; cf. Dennefeld \& Kunth 1981; Blair, Kirshner, $\&$ Chevalier 1981) concerning both the absolute values and the shape. Although radial distributions of abundances are often referred to as logarithmic "abundance gradients" in the literature, evidencing the exponential nature of the distributions, it should be recalled that there is no observational evidence for a single slope of this gradient, since, for the reasons given in $\S 2$, i.e., the lack of observations of $\mathrm{H}$ II regions near the central regions of galaxies and the difficulty of obtaining accurate abundances for the inner regions, our description of the metallicity distributions in spiral galaxies is rather incomplete. Actually, in the few cases for which a good sampling exists (e.g., M33; Vílchez et al. 1988), the single-slope hypothesis breaks down. Other works also point to the presence of more general abundance radial distributions in spiral galaxies (Díaz 1989; Zaritsky 1992). Thus, it may be completely misleading to speak of a "gradient," which is inaccurate from both a mathematical point of view and the physical interpretation of the data. It is the actual data that are to be fitted, and not their approximate representation by a single-parameter curve.

In Figure $2 b$, we plot the radial distribution of the ratio $\mathrm{N} / \mathrm{O}$. This type of figure, which shows the relative abundance between two elements, gives us information about the 

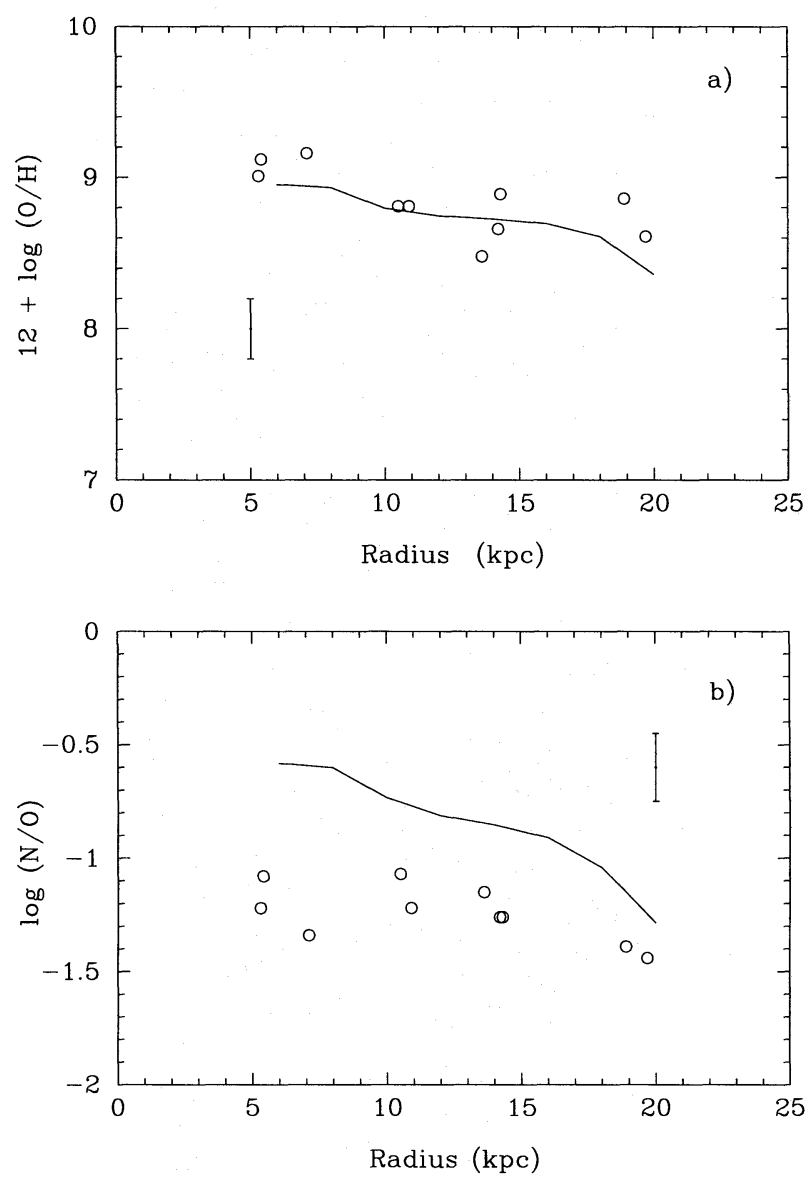

Fig. 2.-Radial distributions of abundances for NGC 224: (a) $12+\log (\mathrm{O} / \mathrm{H}) ;(b) \log (\mathrm{N} / \mathrm{O})$. Data are from Dennefeld \& Kunth (1981) and from Blair et al. (1981).

nucleosynthetic processes. If one of them, in this case oxygen, is well fitted but $[\mathrm{N} / \mathrm{O}]$ is higher than observed, it may indicate either that a stellar yield with more primary or secondary nitrogen than real was used, or that an IMF biased to low-mass or massive stars was used. This effect is seen in all galaxies. The nucleosynthetic yield of nitrogen has been taken from the rather old study by Renzini \& Voli (1981); there are recent developments in this field (Thielemann, Nomoto, \& Hashimoto 1994) that may change this yield, as was discussed regarding the magnesium yield in Paper V.

The time evolution of $[\mathrm{Fe} / \mathrm{H}]$, the so-called agemetallicity relation (AMR), is presented in Figure 3 for three different regions: "inner" $(R=6 \mathrm{kpc})$, "middle" $(R=12$ $\mathrm{kpc})$, and " outer" $(R=20 \mathrm{kpc})$. It is evident from this figure that a unique AMR is not expected in external galaxies. It has been demonstrated for the Milky Way by Edvardsson et al. (1993) that it is impossible to assume a unique AMR: their data present a large intrinsic scatter that can only be ascribed to the overlap of stellar populations. The parallel evolution of halo, thick disk, and thin disk (Paper IV) and the parallel evolution of nearby rings naturally produce the observed dispersion. The multiphase model predicts a similar behavior in external spiral galaxies.

The effect of the creation of a molecular ring in the range $8-14$ kpc (Fig. $1 b$ ) is evident in the distribution of the recent star formation (Fig. 4), which follows the cloud distribution. In our models, it appears that the molecular ring is formed by the balance between accumulation of gas from the halo,

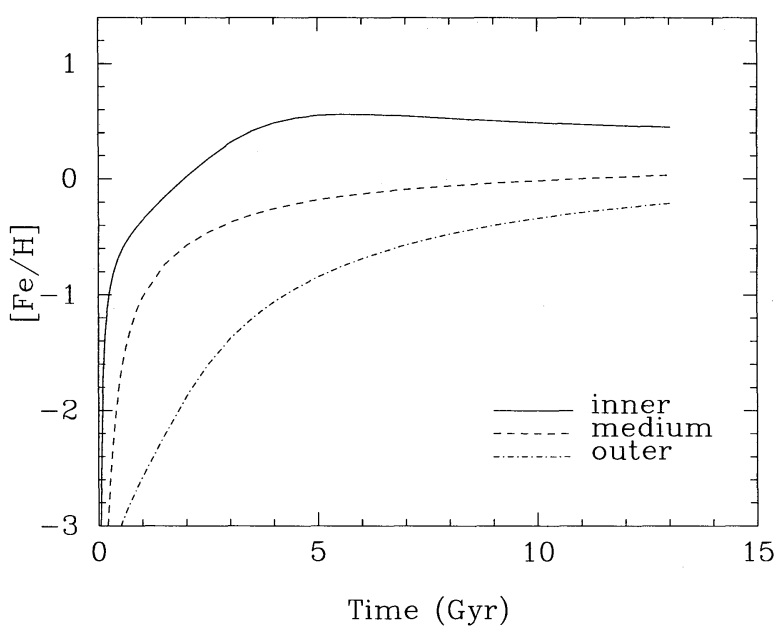

Fig. 3.-Age-metallicity relation for three different zones of NGC 224: inner $(R=6 \mathrm{kpc})$, medium $(R=12 \mathrm{kpc})$, and outer $(R=20 \mathrm{kpc})$.

gas conversion to clouds, and cloud destruction. The temporal history of star formation in the various regions is such that in the internal regions a large amount of gas was able to produce a large reservoir of clouds that efficiently fed the star formation; after a very active phase, the SFR was strongly reduced as a result of a scarcity of fuel. In the intermediate region, in the middle of the molecular ring, the star formation has varied only by a factor of 2 from the maximum to the present. This region probably corresponds to the solar neighborhood (Paper II); the self-regulation in a reasonably fed zone maintains the star formation for long times. In this case, the timescale for gas consumption cannot simply be evaluated from an exponential decrease of the present gas fraction with a star formation timescale, as has often been discussed in the literature. In zones at large galactocentric distances, the evolution is still lacking, because of a less efficient accumulation of gas, a reduced capability to form clouds from diffuse gas $[\mu(R)$ decreases with distance], and a lower frequency of cloud collisions, because of both geometrical dilution and dynamical reasons $[H(R)$ decreases]. This complexity of physical effects has been interpreted in the literature as evidence for the existence of a threshold in star formation (Kennicutt 1989). In fact, the multiphase model has a behavior very similar to Kennicutt's model, which is evident from the relation

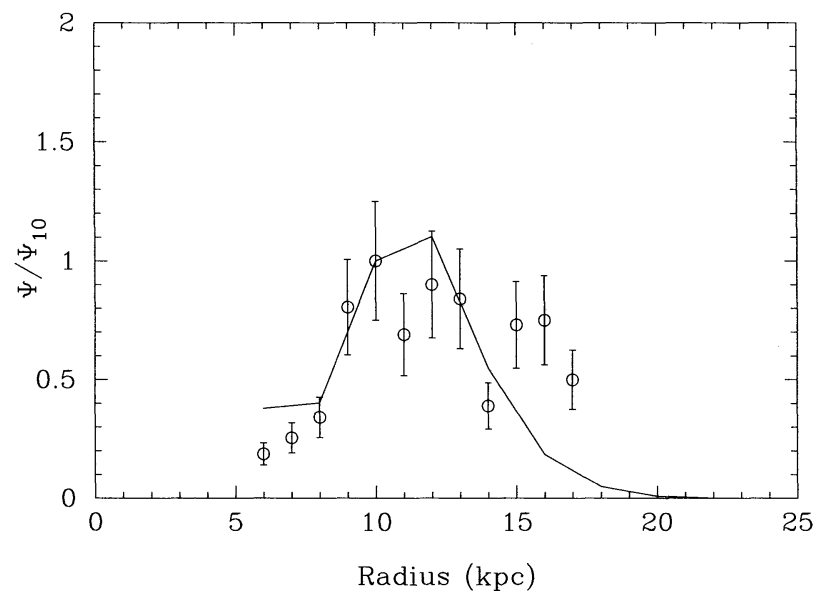

FIG. 4.-Star formation rate surface density $(\Psi)$ in NGC 224 , normalized to $\Psi$ at the equivalent solar radius $\Psi_{10}$. Data are from Walterbos \& Braun (1992). 
between the SFR surface density and the gas surface density. This point is further discussed in $\S 5$ (Fig. 20, below). However, the steep exponential form usually obtained from observations for the SFR radial distribution is not reproduced by the model results, which show a flatter distribution.

In summary, the model fits most observational constraints and provides a theoretical interpretation of the conclusions reached from the observations by other authors about NGC 224. It results in a galaxy more metal-rich than ours, more evolved, with a smaller number of molecular clouds as compared with the total mass of gas, and an SFR that has been higher in the past.

\subsection{NGC 300}

Only data for the diffuse gas are available for comparison with the model predictions for NGC 300 . This galaxy shows an interesting evolutionary history - the collapse timescale is longer because of its small total mass, and the adopted efficiencies are lower because of its late Hubble type. These two facts combine to produce a slower evolution, which is apparent in all of the model results. The atomic and molecular hydrogen radial distributions have peculiar evolutions with respect to the Milky Way since the collapse of diffuse gas from the halo to the disk is more efficient than its conversion into clouds (Fig. 5). The observed $\mathrm{H}$ I distribution is almost perfectly reproduced by the model, with a maximum at $R=3-4 \mathrm{kpc}$, near the observed one.
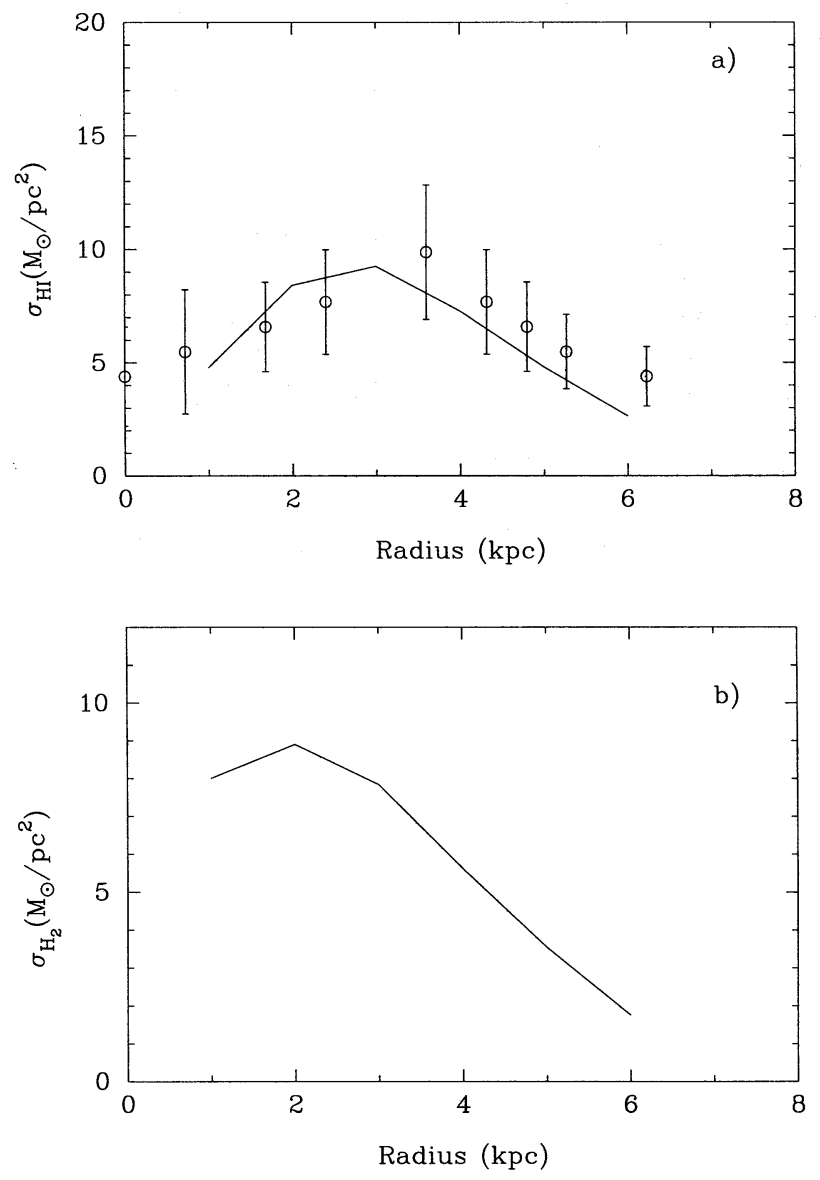

FIG. 5.-Radial distributions of gas surface densities for NGC 300: (a) diffuse gas or atomic component and (b) molecular clouds. Data are from Rogstad, Crutcher, \& Chu (1979).
The observed radial distribution of oxygen (Fig. 6) is adequately reproduced and appears morphologically different from the one found for NGC 224. Comments about the nitrogen yield are the same, as a result of the similar behavior in the $[\mathrm{N} / \mathrm{O}]$ versus oxygen abundance relation.

The computed iron abundance tells us that this is a metal-poor galaxy since no ring reaches solar metallicity; the three disk regions show the same behavior as the outer regions of the more massive galaxies, the most important point being the rather low growth of iron enrichment in the early stages of evolution; the halo shows a low iron abundance as well.

The SFR, shown in Figure 7, is almost an exponentially decreasing function of radius, except for the innermost disk. The maximum at $R \sim 2 \mathrm{kpc}$ with a plateau between 1 and 2 $\mathrm{kpc}$ was observed in this region by Deharveng et al. (1988), both in the $\mathrm{H} \alpha$ flux and for the number of $\mathrm{H}$ II regions. The case of NGC 300 suggests some reflections on the approximations made in the model. The rather low increase of metal abundances with time, due to inefficient cloud and star formation, may have a feedback effect: $\mu$ is a complicated function of the thermal, gravitational, and magnetic conditions of the gas (Shore \& Ferrini 1995). It depends on gas temperature and hence must be a function of $Z(t)$; lower $Z$ would correspond to lower $\mu$ and to a reduced conversion rate from diffuse gas to clouds. This produces lower star formation at early stages and higher star formation later on; a factor of $\sim 2$ for the present star formation rate in the internal regions is not unexpected.
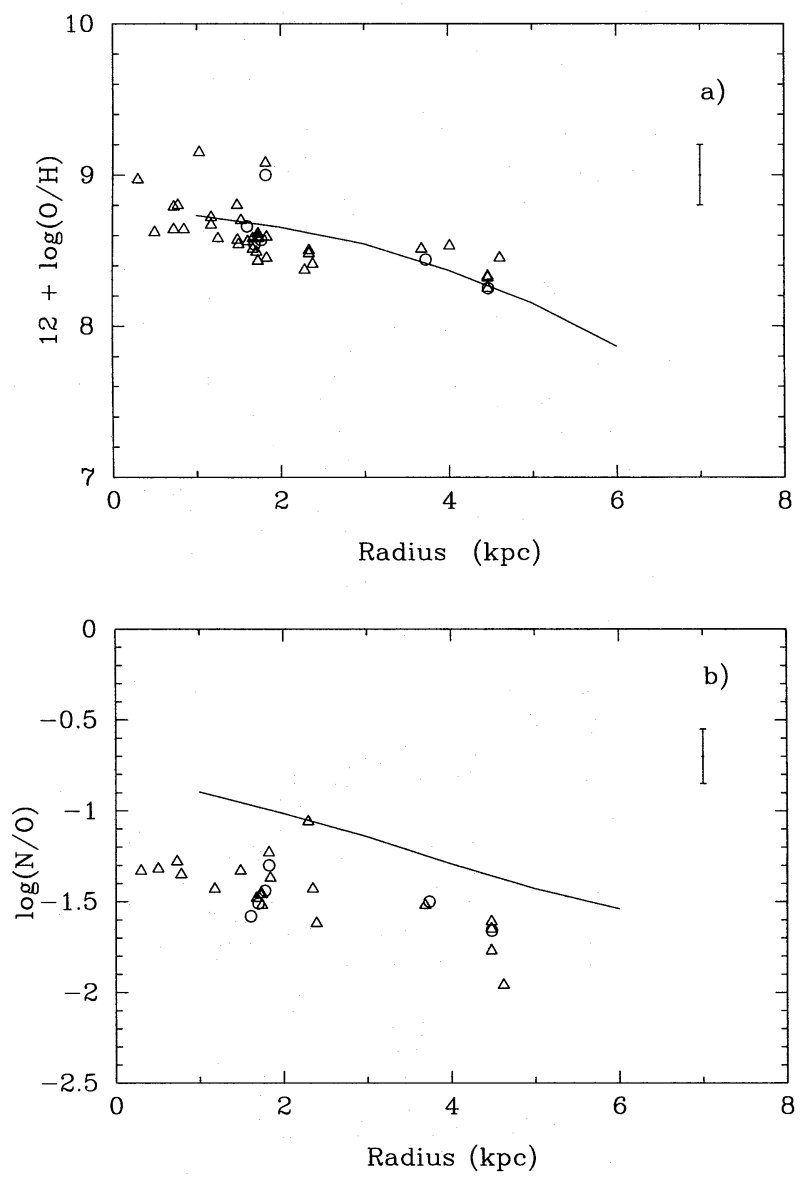

Fig. 6.-Same as Fig. 2, but for NGC 300. Data are from Pagel et al. (1979; circles) and from Deharveng et al. (1988; triangles). 


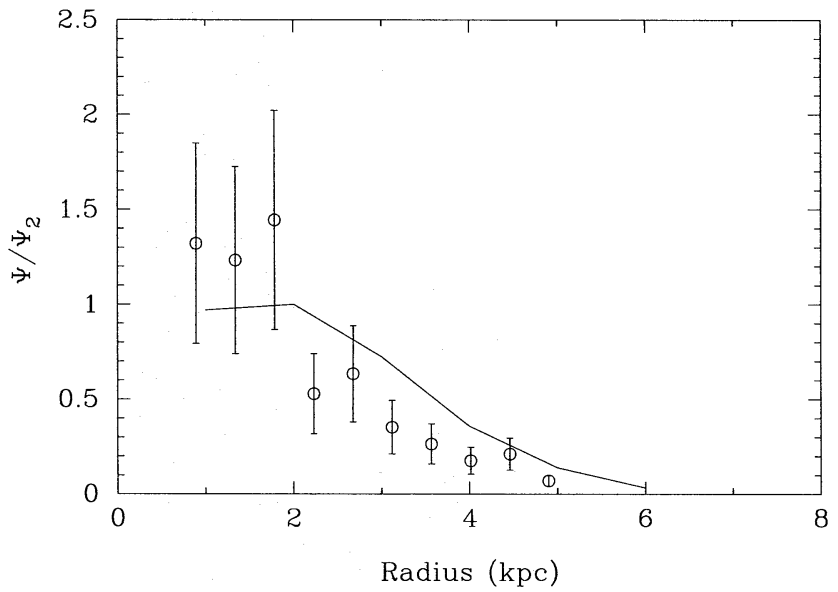

FIG. 7.-Star formation rate surface density in NGC 300, normalized to $\Psi_{2}$. Data are from Deharveng et al. (1988).

\subsection{NGC 598}

A good agreement between model predictions and observations is found for NGC 598. The present-day neutral gas radial distribution, plotted in Figure $8 a$, agrees with the data within the errors; the same occurs for the molecular gas distribution, shown in Figure $8 b$.

The abundance radial distribution of oxygen, shown in Figure 9, is well reproduced; the present-day SFR distribution is in excellent agreement with the observations (Fig. 10). The AMR is similar to that of NGC 300, with the
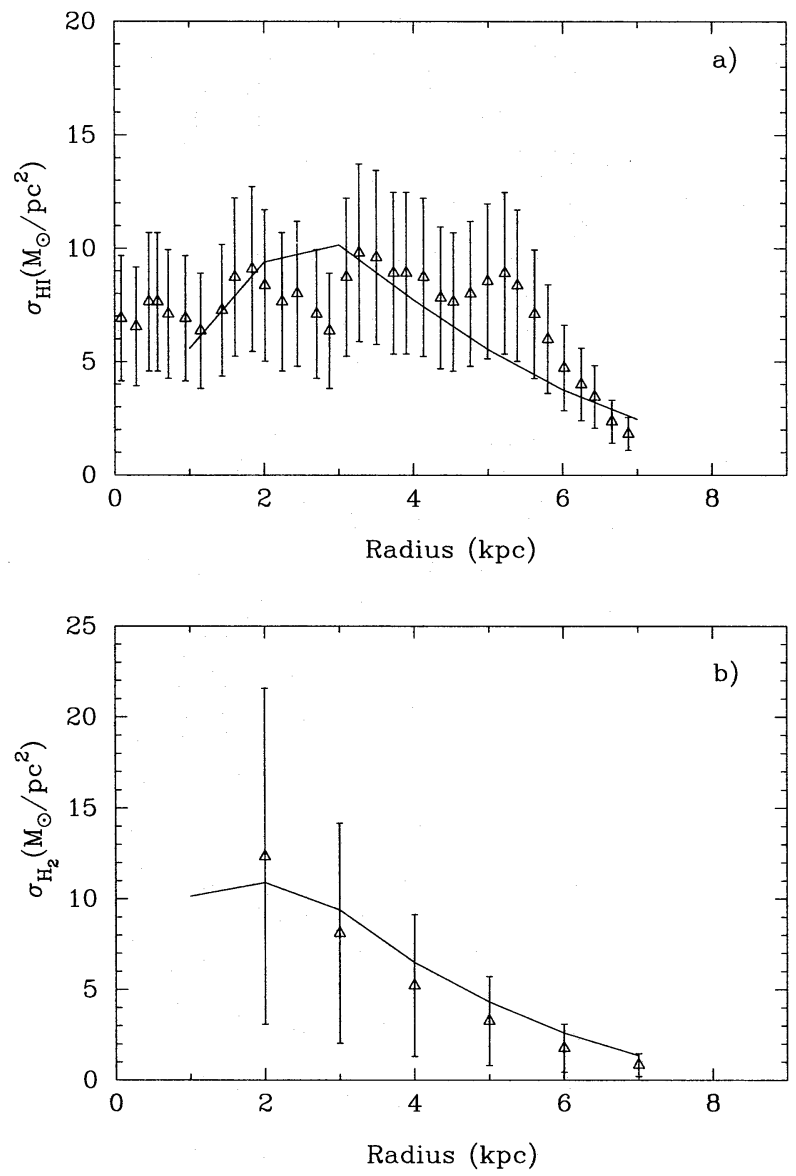

Fig. 8.-Same as Fig. 5, but for NGC 598. Data are from Newton (1980) for $(a)$ and from Tosi \& Díaz 1985 for $(b)$.
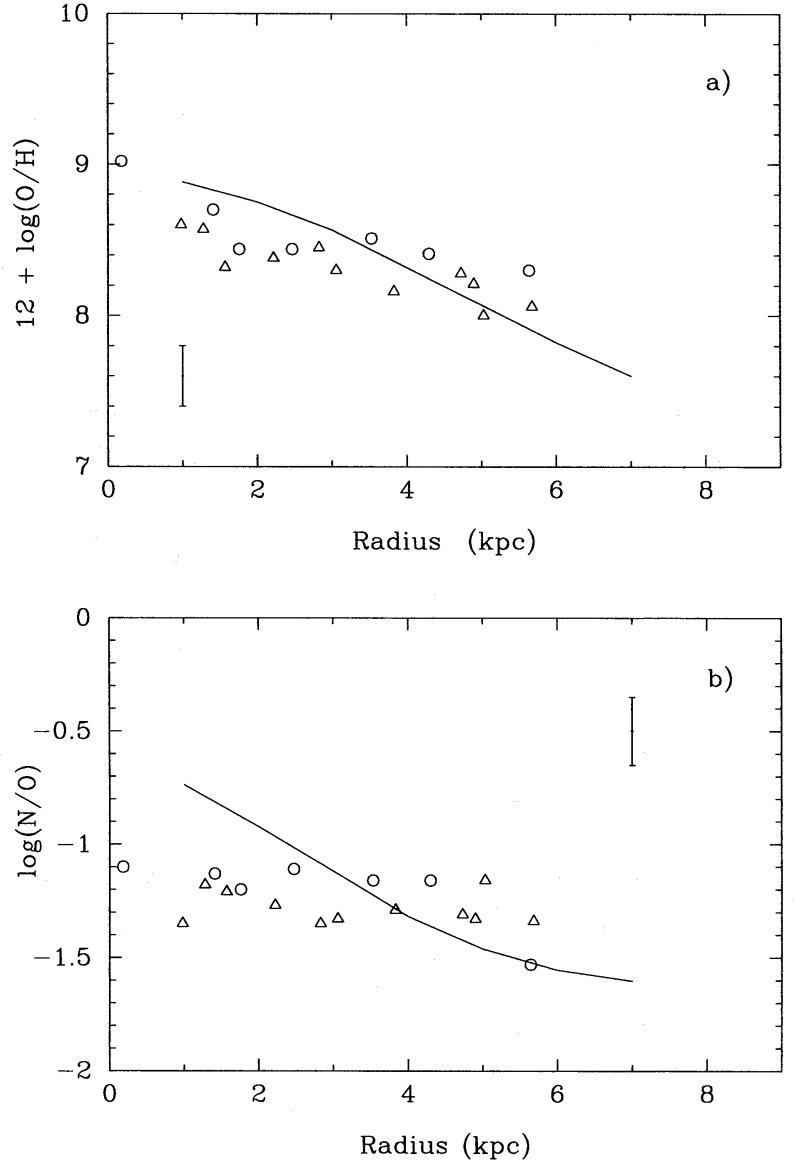

Fig. 9.-Same as Fig. 2, but for NGC 598. Data are from Kwitter \& Aller (1981; triangles) and from Vílchez et al. (1988; circles).

exception of the inner region $(R \leq 1 \mathrm{kpc})$, which reached solar metallicity at $10 \mathrm{Gyr}$.

The usual way that gradients are determined, namely, least-squares fitting of logarithmic abundances to a single straight line, can in principle be questioned. It implies an exponential radial distribution of abundances, which in some cases might not be an adequate representation of reality (e.g., M33; see Vílchez et al. 1988).

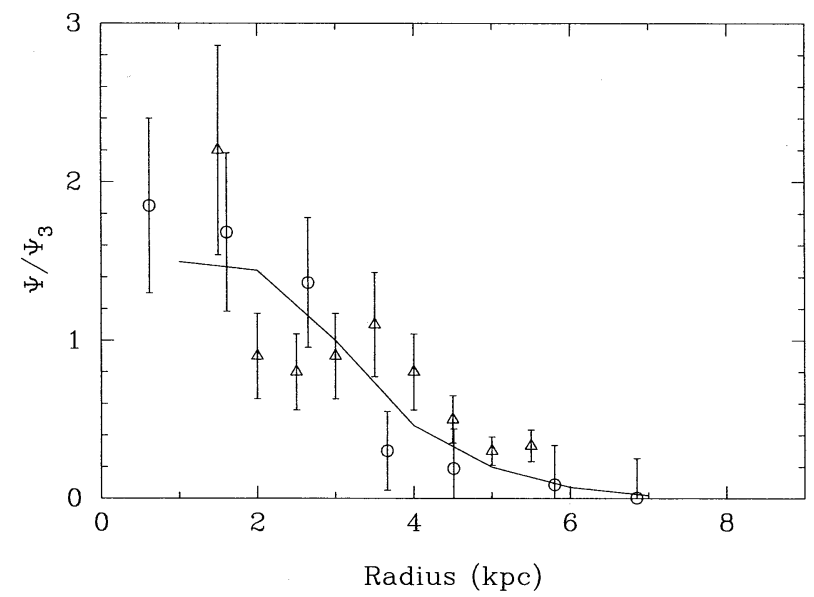

Fig. 10.-Star formation rate surface density for NGC 598, normalized to $\Psi_{3}$. Data are from Kennicutt $(1989$; circles) and from Rumstay \& Kaufman (1983; triangles). 


\section{4. $N G C 628$}

There is no particular comment to be made from the comparison of observations of NGC 628 with the model (Figs. 11, 12, 13) but for the decrease of star formation activity in the model for small galactocentric radii, which is not apparent in the data. It is difficult to deduce any indication from this disagreement; it may be real or a consequence of the observational difficulties involved in the reconstruction of a correct SFR estimate for the central region.

However, the computed SFR follows, as in the other galaxies, the molecular gas surface density distribution. It is difficult to compare it with the observed one because of the large differences between data from different authors, but a recent CO map of the inner spiral arms from Wakker \& Adler (1995) seems to indicate that there is no concentration of $\mathrm{CO}$ in the inner region. These authors suggest the possibility of $\mathrm{CO}$ deficiencies where central peaks were believed to exist. If this "hole" is real, in agreement with the model results of Figure $11 b$, the decrease of the SFR in the inner region could be real, too.

A decrease in the radial distribution of SFR surface density or molecular gas surface density at small galactocentric radii may be due to the bulge's influence on the disk; as a consequence, this zone, close to the bulge, must be treated in a way different from the disk (see Paper V). It should be remembered that our model only applies to the disk and to the region of the halo below and above the disk, with the bulge being out of this calculation.
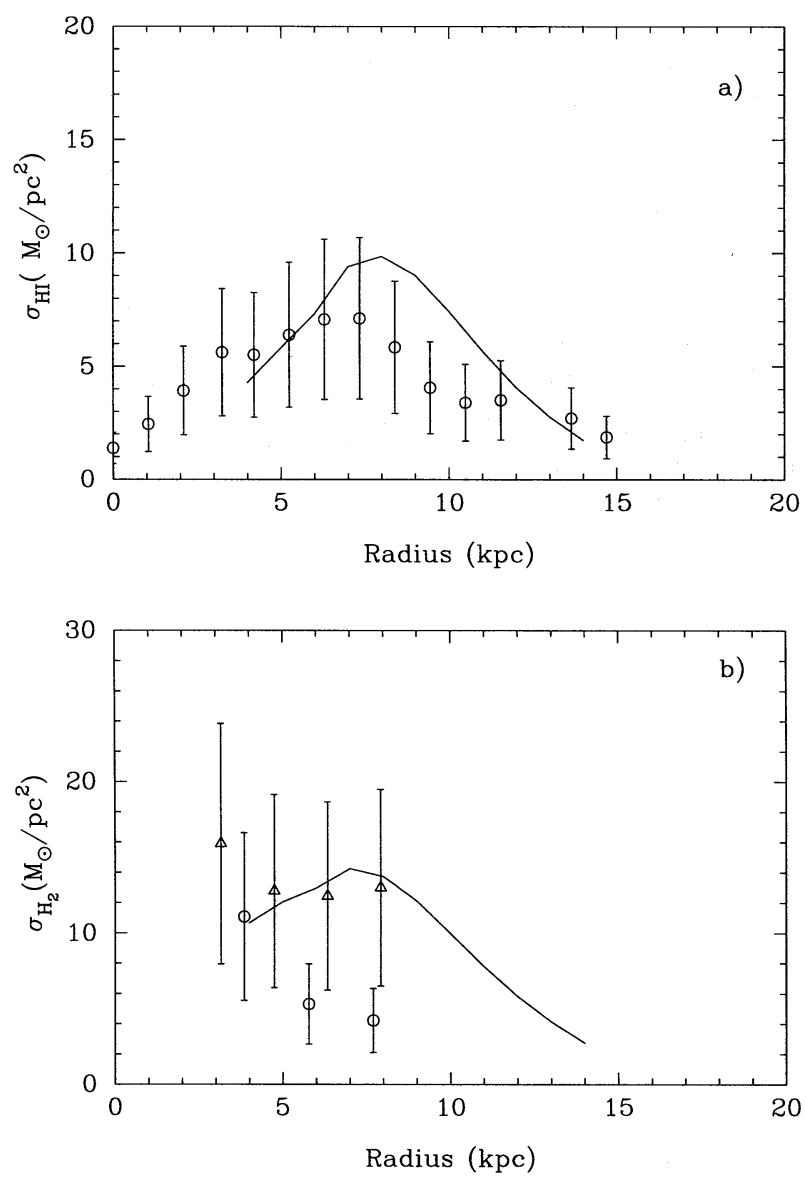

Fig. 11.-Same as Fig. 5, but for NGC 628. Data are from Wevers, van der Kruit, \& Allen (1986) for (a) and from Sage (1993; circles) and Adler \& Liszt (1989; triangles) for (b).
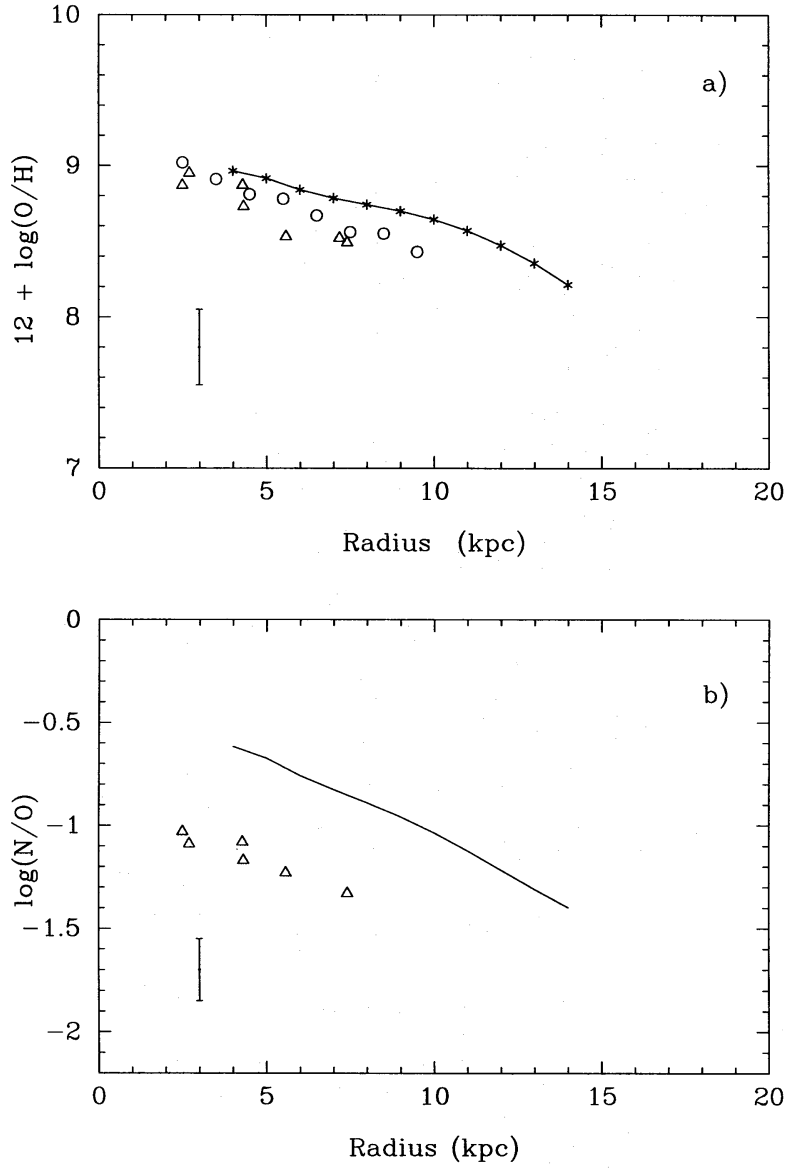

Fig. 12.-Same as Fig. 2, but for NGC 628. Data are from McCall et al. (1985; triangles) and from Belley \& Roy (1992; circles).

Alternatively, the accumulating gas could last longer, with the assumption of a slower infall rate (larger collapse time) or a larger initial mass.

\subsection{NGC 3198}

The model results for the well-studied galaxy NGC 3198 are rather successful, including a correct prediction for the halo-to-disk mass ratio. It would be interesting to compare other predictions (Figs. 14, 15, 16) of our model (such as the molecular gas densities and the other elements' distributions) with future observations. It would be impor-

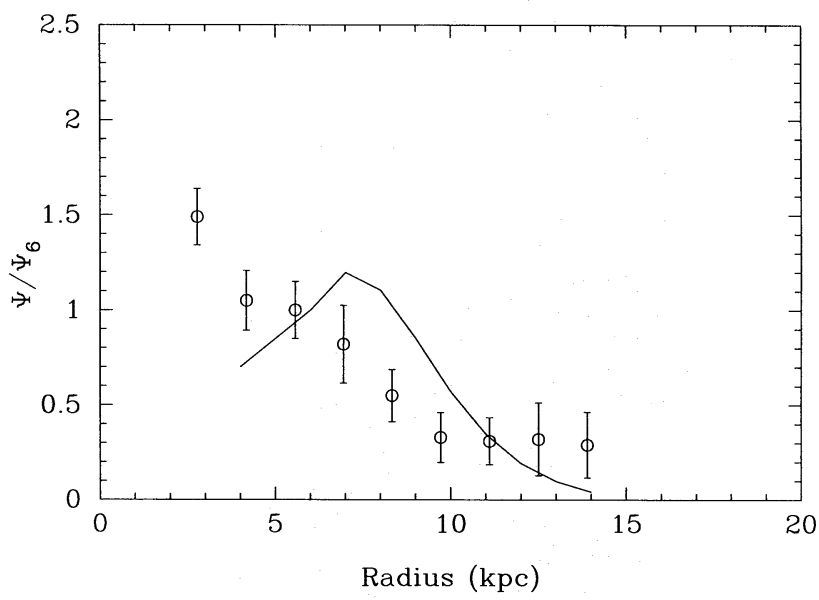

FIG. 13.-Star formation rate surface density for NGC 628, normalized to $\Psi_{6}$; data are from Natali, Pedichini, \& Righini (1992). 

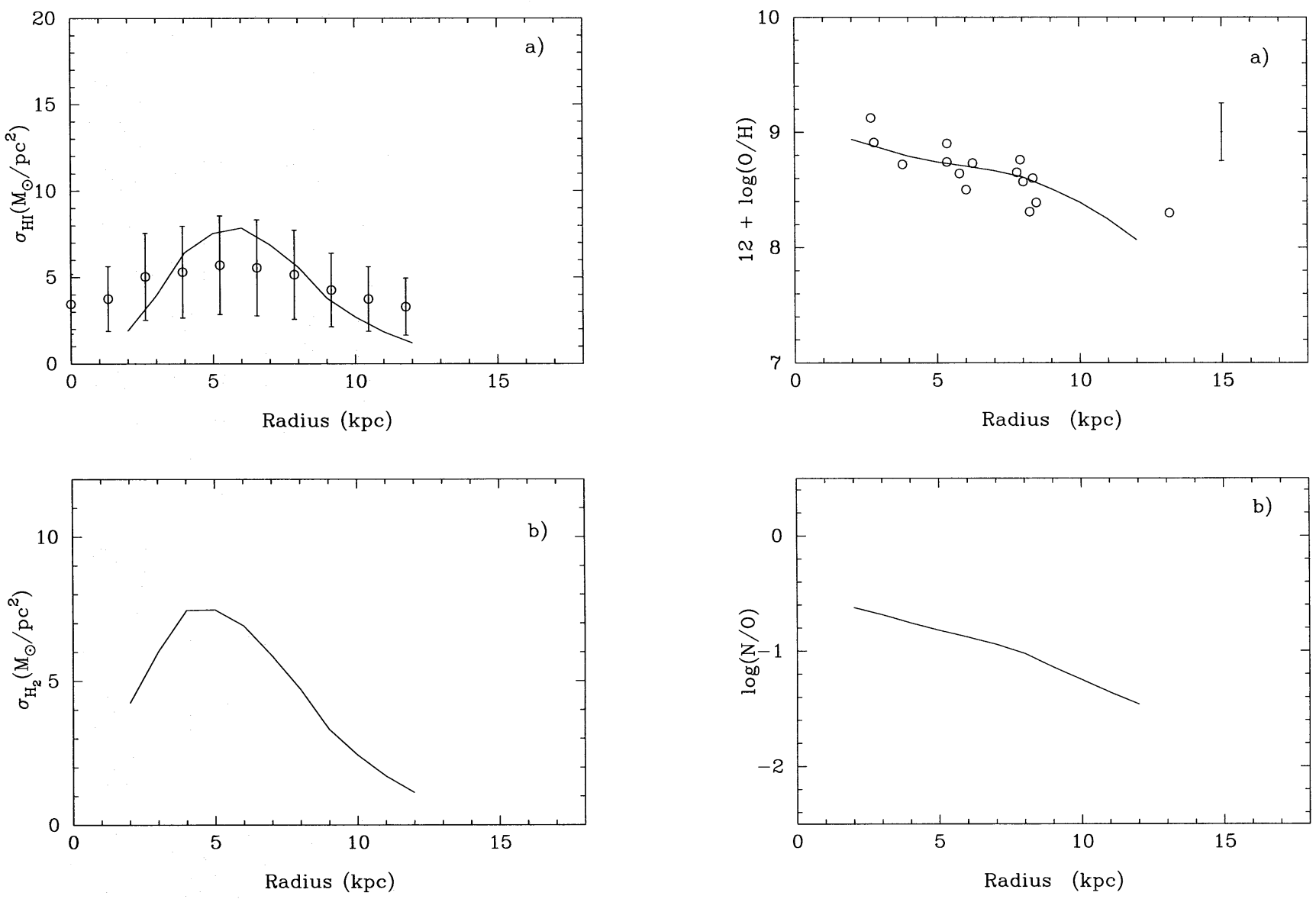

Fig. 14.-Same as Fig. 5, but for NGC 3198. Data are from Wevers et al. (1986).

tant to decide if the multiphase approach and Schmidt-law bookkeeping are equally able to describe galactic evolution.

\section{6. $N G C 6946$}

For NGC 6946, we have a very complete set of data for the radial distributions of gas, abundances, and SFR, allowing a good comparison between model results and observations.

The predicted atomic gas amount agrees well with that observed (Fig. 17a). There is also good agreement in the shape of the gas distribution, with a maximum at the 5-6 $\mathrm{kpc}$ region. For the molecular gas distributions (Fig. 17b), the same comments made in the case of NGC 628 apply. Figure 18 shows the good agreement between observed and model-predicted oxygen abundances. Finally, in Figure 19 the SFR surface density normalized to the $R=5 \mathrm{kpc}$ region's value $\left(\Psi_{5}\right)$ presents a behavior in excellent agreement with observations, with the inner regions showing the same behavior as found for NGC 628 .

\section{DISCUSSION}

\subsection{Abundance Radial Distributions: Correlations with Morphological Type}

The existence of abundance radial gradients in the disks of spiral galaxies has been the object of numerous studies, developed, in general, with the aim of finding systematic behavior. From the observational point of view, correlations have been sought between absolute values of abundances and/or their gradients and other properties of

Fig. 15.-Same as Fig. 2, but for NGC 3198. Data are from Zaritsky et al. (1994).

galaxies: morphological type, total mass, bolometric luminosity, and gas fraction. Two recent studies following this scheme are those of Vila-Costas \& Edmunds (1992) and Zaritsky et al. (1994). The results of these two works can be summarized as follows:

1. There is a correlation between the central value of the metallicity, as extrapolated from the obtained gradient, and the morphological type of a galaxy: early-type galaxies have larger central abundances than late-type ones. The same is

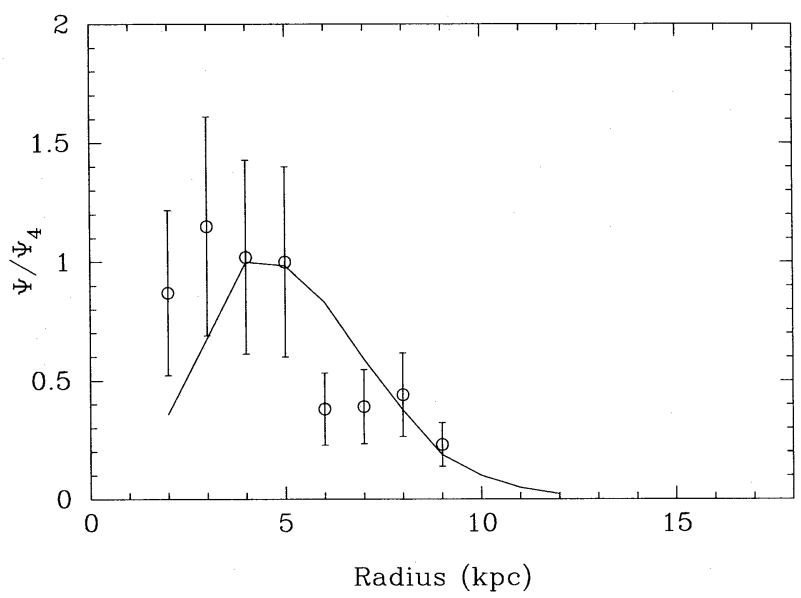

FIG. 16.-Star formation rate surface density for NGC 3198, normalized to $\Psi_{4}$. Data are from Corradi et al. (1991). 

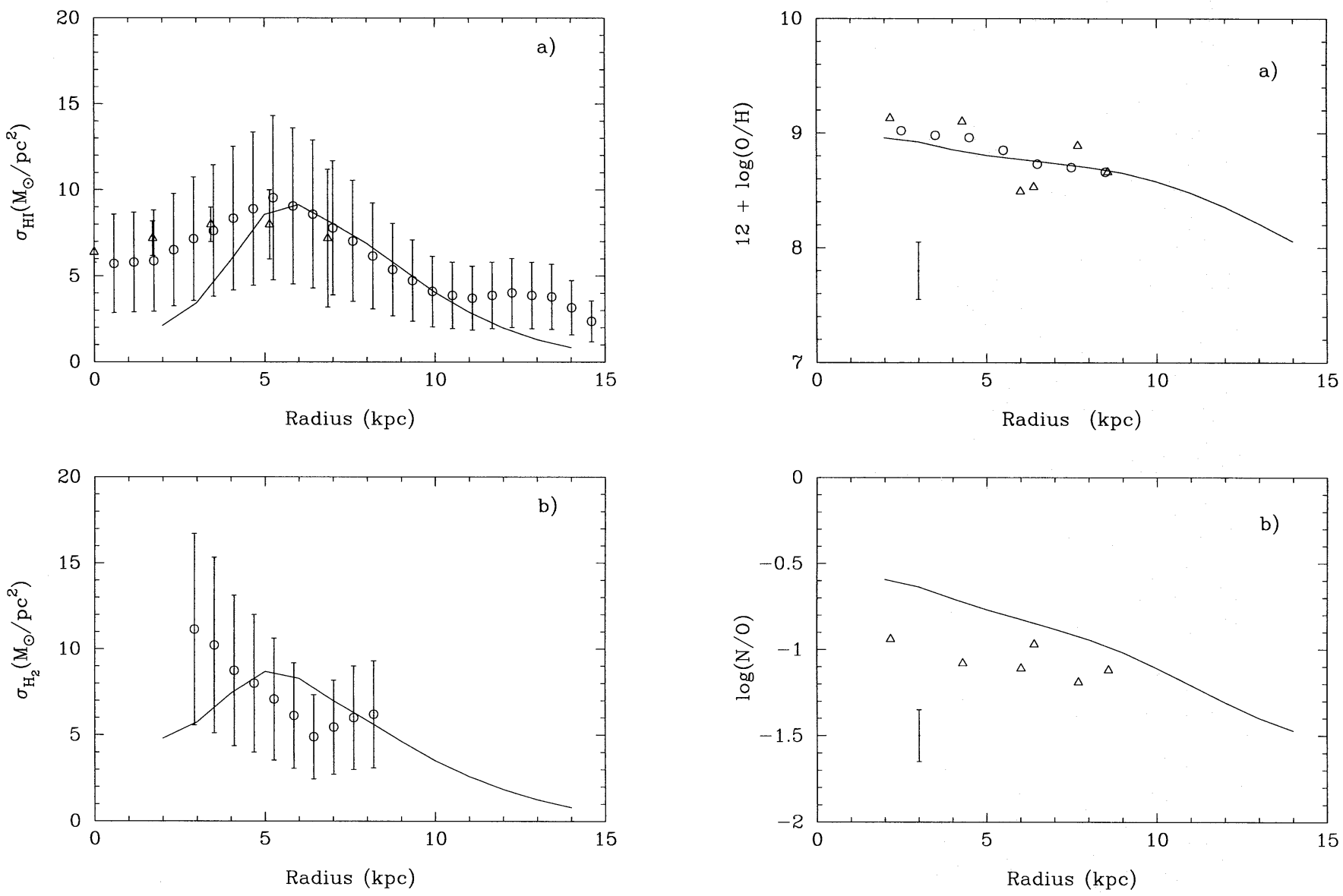

Fig. 17.-Same as Fig. 5, but for NGC 6946. Data are from Tacconi \& Young (1986).

found for the characteristic disk abundance, defined as the abundance at a fixed radius (different for each author), which also correlates with the total mass of the galaxy.

2. There is a correlation between the radial gradient (in dex $\mathrm{kpc}^{-1}$ ) and morphological type: late-type galaxies show a steeper gradient than early-type ones. This had already been suggested by Pagel (1991) and is supported by results from Oey \& Kennicutt (1993) that, although with a large uncertainty, indicate flatter gradients for early-type galaxies. However, if the radius is normalized to the isophotal radius, the correlation disappears, implying a relation between the existence of the gradient and the actual size of the disk.

3. Barred galaxies have shallow gradients. This result has been confirmed by Edmunds \& Roy (1993) and Martin \& Roy (1994), the latter of whom claimed that this results seems to indicate that the present bar induces a large-scale mixing of the interstellar medium, in agreement with recent results of radial flow models. Friedli, Benz, \& Kennicutt (1994) suggested, along this same line, that early galaxies with flat abundance distributions should have or have had in the past an undetected bar.

4. There is a correlation between abundances and mass surface density, which is also related to the morphological type. This correlation can be obtained by using the total mass surface density or only the corresponding exponential disk surface density (Ryder 1995). For late-type spiral galaxies there is no difference between the two methods, but for

early-type ones this difference is large, as a result of the presence of the bulge; the correlation is stronger if the total mass is used.

5. A plot of the existing oxygen abundances of $\mathrm{H}$ II regions in spiral galaxies, taken together, versus their positions in the disks of their parent galaxies shows a large

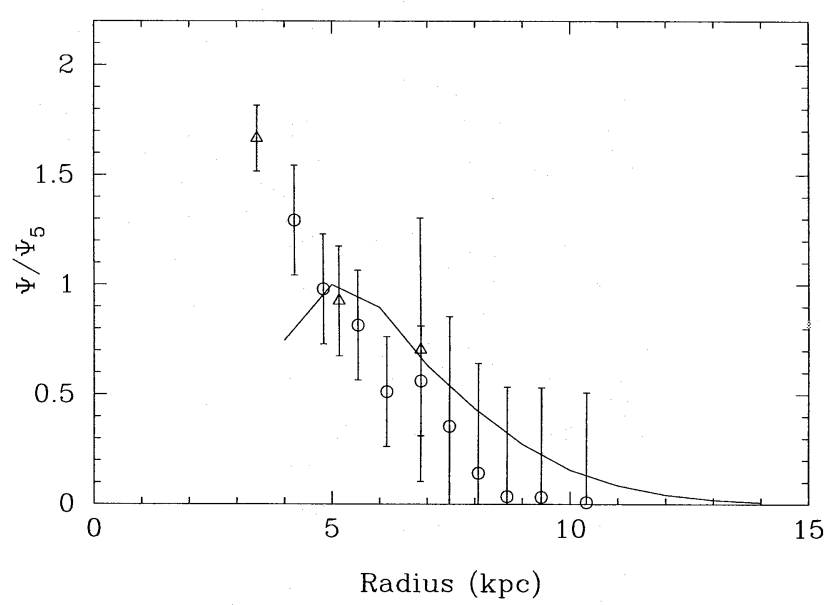

FIG. 19.-Star formation rate surface density for NGC 6946, normalized for the same quantity in the equivalent to solar region $\Psi_{5}$; data are from DeGioia-Eastwood et al. (1984; triangles) and from Kennicutt (1989; circles). 
dispersion that does not disappear when a radial normalization with respect to the isophotal or effective radius is made. The scatter is partially a result of the fact that different galaxies have different average metallicities, and it therefore is greatly reduced if abundances are also normalized to a reference value for each galaxy, e.g., the abundance corresponding to the effective radius of its disk. Using this representation, Díaz (1989) found a linear relation between the logarithmic oxygen abundance and the galactic radius of the form $\log (\mathrm{O} / \mathrm{H})-\log (\mathrm{O} / \mathrm{H})_{R_{\text {eff }}}=a-b\left(R / R_{\text {eff }}\right)$ for $R \leq 1.50 R_{\text {eff }}$, where $R_{\text {eff }}$ is the photometric effective radius of the disk, with the distribution being rather flat for larger radii. This relation implies that when $R_{\text {eff }}$ is smaller, the gradient $b / R_{\text {eff }}$, in dex $\mathrm{kpc}^{-1}$, is steeper. Because of the smaller effective radius for late-type spiral galaxies (see Table 4 of Simien \& de Vaucouleurs 1986), which corresponds to a lower value for the luminous matter, this leads to the same conclusion reached in point 2.

6 . There exists a correlation between the atomic gas fraction and the oxygen abundance, as suggested by Pagel (1991). If the gas molecular component is included, the correlation remains only if the conversion factor $\chi$ of the $\mathrm{CO}$ intensity to molecular hydrogen density is not the same for every galaxy, so that a smooth radial distribution of total gas is obtained.

7. There is a correlation between the oxygen abundance and the gas fraction in molecular form $\left[\sigma\left(\mathrm{H}_{2}\right) / \sigma_{\mathrm{gas}}\right]$, as suggested by Tosi \& Díaz (1990).

Our models predict steeper radial distributions for latetype galaxies as a consequence of the smaller efficiencies and slower collapse in these galaxies. In most cases, the observed abundances and abundance gradients are well reproduced. The computed abundance radial distributions flatten with time as a consequence of the evolution of the galaxy. The time evolution of gradients is a delicate, important point in itself, and a severe test for models; therefore, it will be addressed in a forthcoming paper. Only some general comments are made here. The gradient seems to be a differential effect between regions that evolve at different rates, reaching a saturation level at a different times. The inner regions of the disks reach this level in a short time, and a steep gradient is promptly developed. When the outer regions also reach their maximum abundance level, the gradient becomes shallower and even disappears in some cases. Therefore, if a given galaxy is in an early evolutionary state, the radial distribution is still steep. The earlier morphological type galaxies are more massive and therefore collapse much more rapidly, showing a faster evolution and a higher SFR. Because of this, the saturation level is reached rapidly. Thus the first two results of Vila-Costas \& Edmunds (1992) and Oey \& Kennicutt (1993) listed above are reproduced by our model-predicted results and arise naturally from the physics underlying our theoretical models.

A possible reason for the existence of gradients is the radial variation of the SFR. In our models, this variation is due first to the radial dependence of the model parameters, including the infall rate, when volume effects are introduced, and secondly to the radial dependence of the efficiency $\epsilon_{\mu}$, included in the model to take into account the frequency of the spiral wave's passage, which is higher in the inner regions than in the outer ones. If the spiral wave did not exist, the radial gradient would not appear or would be flatter, and if the infall rate is high, an accumulation of mass like a bar may be induced in the center, leading to a quick evolution that produces a flat abundance radial gradient. This last effect may be responsible for the lack of a gradient in barred galaxies (see point 3 above).

\subsection{Correlations between Gas Fraction and Morphological Type}

Several sets of observations provide information about the correlations between the molecular gas fraction $\left[M\left(\mathrm{H}_{2}\right) / M(\mathrm{H})\right]$ and morphological type (see, e.g., Young \& Knezek 1989). There is marginal evidence that this ratio increases from late-type spiral galaxies to early types. This increase could be interpreted as a higher efficiency to form molecular clouds per atomic mass unit in early-type galaxies than in late types.

Model results (Table 5) also suggest an approximate positive correlation in the relative importance of molecular to atomic gas with type, although the relationship is not strictly monotonic. A stronger correlation is found if we compare the values corresponding to different arm classes according to the classification proposed by Elmegreen \& Elmegreen (1987): the increase of the ratio is now monotonic from class 5 ("two symmetric, short arms in the inner regions; irregular outer arms") to class 9 ("two symmetric inner arms; multiple long and continuous outer arms").

Turning to the ratio of total gas mass to dynamical mass of the galaxy, the classic studies suggest an increase of the gas fraction with the type (see, e.g., Young \& Scoville 1991). The models reproduce this trend, which is again clearer if we consider the arm class classification. The reason for the very well defined correlation of the gaseous fractions with

TABLE 5

RESULTS FROM MODELS

\begin{tabular}{|c|c|c|c|c|c|c|}
\hline Galaxy & Type & $\log \mu^{\mathrm{a}, \mathrm{b}}$ & $m\left(\mathrm{H}_{2}\right) / m\left(\mathrm{H} \mathrm{I}^{\mathbf{b}}\right.$ & $\begin{array}{l}d[\mathrm{O} / \mathrm{H}] / d R^{\mathrm{c}} \\
\left(\operatorname{dex~} \mathrm{kpc}^{-1}\right)\end{array}$ & $\mathrm{O}_{c}^{\mathrm{d}}$ & $m_{\mathrm{halo}} / m_{\mathrm{disk}} \mathrm{b}$ \\
\hline Milky Way...... & 4 & -1.31 & 0.46 & -0.08 & 9.00 & 0.15 \\
\hline NGC $224 \ldots \ldots \ldots$ & 3 & -1.60 & 0.45 & -0.025 & 9.09 & 0.16 \\
\hline NGC $300 \ldots . . .$. & 7 & -0.50 & 0.86 & -0.183 & 8.89 & 0.92 \\
\hline NGC $598 \ldots . . .$. & 6 & -0.39 & 0.88 & -0.209 & 8.97 & 0.33 \\
\hline NGC $628 \ldots . . .$. & 5 & -1.52 & 1.51 & -0.090 & 9.18 & 0.07 \\
\hline NGC $3198 \ldots \ldots$ & 5 & -0.77 & 0.96 & -0.111 & 9.00 & 0.33 \\
\hline NGC $6946 \ldots . .$. & 6 & -0.94 & 0.96 & -0.085 & 9.24 & 0.55 \\
\hline
\end{tabular}

${ }^{\text {a }}$ Gas fraction measured by $\log \left(m_{\mathrm{gas}} / m_{\mathrm{tot}}\right)$.

b Values are for the equivalent solar radius $R_{0}$.

c Radial gradient value for oxygen abundances.

d Central value for the oxygen abundance measured in the scale $12+\log (\mathrm{O} / \mathrm{H})$. 

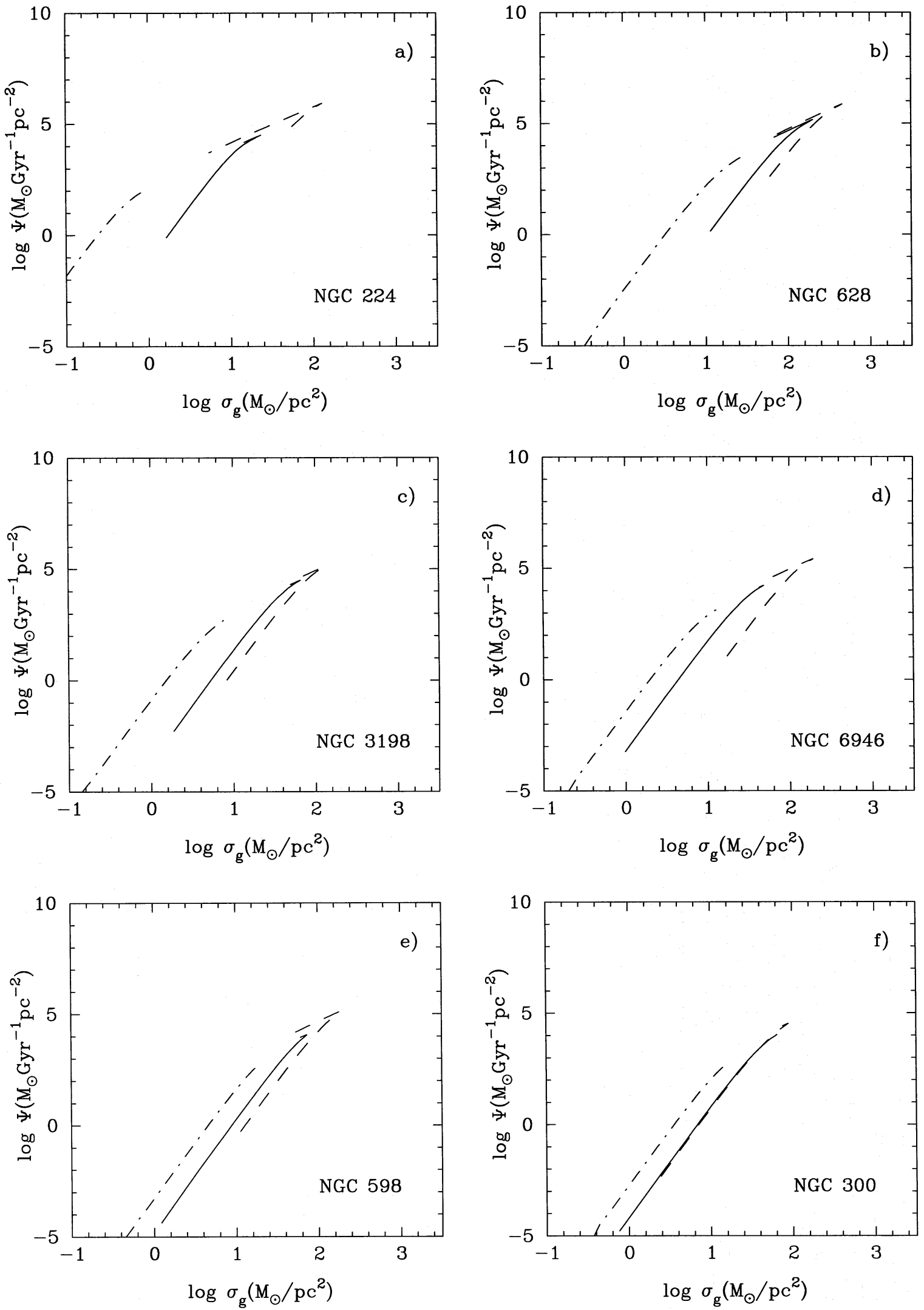

Fig. 20.- Relation between the star formation rate surface density and the total gas surface density for three radii: inner (dashed lines), medium (solid lines), and outer (dot-dashed lines), for the six sample galaxies: (a) NGC 224, (b) NGC 628, (c) NGC 3198, (d) NGC 6946, (e) NGC 598, and ( $f$ ) NGC 300.

arm class is easily understood in our scheme: the cloud formation and the cloud interactions are tightly connected with the properties of the arms, and the fate of the gaseous populations is governed by the arm structure.
We suggest that the morphology of the gas in spiral galaxies could be interpreted according to this concept, which would provide a physical basis for the observed correlations. 


\subsection{Star Formation Rate}

Regarding the SFR, several studies indicate that it was higher in the past for early-type galaxies than for late-type ones where star formation is at present significant (Gallagher et al. 1984; Bothun \& Gregg 1990; Caldwell et al. 1991). The multiphase model also shows SFRs that were higher in the past for early-type galaxies (Ferrini \& Galli 1988), which indeed is one of the reasons they have higher abundances.

Deveraux \& Young (1991), by analyzing the dependence of the far-infrared luminosity on the Hubble type, deduced that the efficiency of massive star formation is very similar for all Hubble types. In our models, the "efficiency" could be evaluated as the ratio of the mass of massive stars to the mass of massive stars and clouds, and this quantity is almost constant in our realizations, in agreement with observations. The self-regulation of the star formation processes leads the system to an almost stationary state, in which this "efficiency" is constant. Obviously, this is a consequence of the previous dynamics of the different phases and cannot be assumed as an input for the evolution.

On the other hand, the relation between the SFR surface density and the total gas surface density computed from our models is similar to that obtained by Kennicutt (1989), assuming a density threshold for the SFR. Figure 20 shows this relation for three radii in every sample galaxy. Two different slopes are apparent. The present-day radii in earlytype galaxies are mostly in the zone where the slope is flatter, while in late-type galaxies most radii are in the zone where this slope is steeper.

\subsection{Shape of the Abundance Distributions}

Perhaps the most important conclusion of our work is that the shape of the radial distribution of the total mass, used as input data for the model, determines the shape of the abundance radial distribution, independent of the efficiencies used, which only produce different absolute values for the abundances. In most cases, the predicted gradient shape is similar to that observed, reproducing not only its value but also its features (one or more slopes for different regions). In fact, if an unrealistic mass distribution is chosen as a model input, it is not possible to reproduce the abundance radial distribution, even if the predicted absolute values are in agreement with the observations. Taking into account that the total mass distribution is the only input data that may have a different radial dependence for every galaxy (the other parameters follow the same radial function included in the model; see Paper III), the importance of this conclusion is clear.

Zaritsky et al. (1994) also analyzed the possible relation between total mass and metallicity $(M-Z)$ in spiral galaxies, concluding that the abundance properties seem to be impressed at an early phase of the galaxies' evolution. Thus the $M-Z$ correlation could actually be measuring the initial structure of a given galaxy. This in turn would determine its Hubble type, thus explaining the correlation between mean abundances and Hubble type. The model also predicts, for the present time, an exponentially decreasing form for the disk mass surface density, similar to those estimated by McCall (1982) from rotation curve decompositions.

Since the total mass is calculated from the rotation curve, this implies a relation between the abundance radial distributions and the rotation curves. These curves are of three different classes: flat, when the rotational velocity is con- stant; increasing, when the rotational velocity increases for increasing radius; and decreasing, when this velocity starts to decrease with radius from a given radius. Forbes \& Whitmore (1989) found a relation between the mass types of spiral galaxies, as derived from these rotation curves, and Hubble type, and it seems that there is also a correlation between the rotation curve gradient and the morphological type (Corradi \& Capaccioli 1990). Taking into account that the gradient of the rotation curve gives us information about the central mass (Baiesi-Pillastrini 1987), the luminosity (Persic \& Salucci 1991), and the quantity of dark mass in the outer halo (Salucci \& Frenk 1989), it is clear that the rotation curve reflects the structural properties of galaxies. Since the bulge-to-disk ratio decreases with the Hubble type (Simien \& de Vaucouleurs 1986), the relation between the central mass, or the corresponding velocity gradient, and the Hubble type is a logical consequence. In this way, it is not strange that a relationship between the mass radial distribution and the abundance gradient exists. Zaritsky (1992) has already suggested the existence of this relation since he found a change of slope in the abundance radial distributions just at the radius where the rotation curve starts to flatten. Using the viscous disk model of Clarke $(1989,1991)$ for the chemical evolution, he concluded that the origin of this flattening is the presence of radial flows of gas, which also makes the abundance gradient flatter. Thus, in the inner regions of spiral disks, where the rotation curve is rising, there are no radial flows, and therefore the abundance gradient may be steeper than in the outer regions, where both the rotation curve and gradients flatten in most spiral galaxies.

Actually, it seems that the initial density, not the total mass, is what produces a given evolution and is responsible for the Hubble type of a galaxy (McGaugh 1994). Thus, galaxies with the same mass may have different types, and there are galaxies with the same type but different luminosity classes or different total masses. In any case, it seems clear that the gravitational collapse and formation of a galaxy (Ryden \& Gunn 1987; Ryden 1988) are related to the chemical features, in particular those of large-scale distributions, apart from local differences that are probably related to density fluctuations.

\section{SUMMARY AND CONCLUSIONS}

We have shown that the multiphase approach to galactic evolution, with a choice of input data consistent with observations, can yield results that are in agreement with available data on the distributions of the elemental abundances, the SFR, and the gas surface density in spiral galaxies of different Hubble types, or, more precisely, of different arm classes.

The input data required by the model are the efficiencies for star formation and molecular cloud formation and destruction, the collapse timescale, and the initial radial mass distribution. The model uses four parameters that consider the relationships determined from the solar neighborhood study (see Papers I-III). These are the efficiencies related to molecular cloud processes $\left(\epsilon_{\mu}, \epsilon_{H}, \epsilon_{a}\right)$ and the collapse time for the accretion of gas from the halo to the disk, $\tau_{\text {coll }}$. The interaction of massive stars with clouds (stimulated star formation) is a local process (see Ferrini \& Galli 1988; Shore \& Ferrini 1995); thus $\epsilon_{a}$ is taken to be the same for every galaxy, and every region within them, as for the Milky Way. The variation of the other two efficiencies 
have been connected to the SDW's action on diffuse gas and clouds. The collapse time is determined by the initial total mass, the mass distribution, and the rotation curve. Therefore, only two efficiencies and the collapse time change from galaxy to galaxy.

The model produces the following:

The radial distributions for atomic and molecular gas; in particular, we obtain different typical radial distributions for both phases-a rather flat distribution for $\mathrm{H}$ I and a ringlike distribution for $\mathrm{H}_{2}$, with an exponential decrease toward large radii.

The ratio of atomic gas mass to molecular gas mass ratio $m_{\mathrm{H}_{\mathrm{I}}} / m_{\mathrm{H}_{2}}$ is correlated with the galaxy classification.

The star formation rates vary with the morphological type as observed, although radial distributions are sometimes flatter than the observed ones in the inner regions of the disks.
Chemical abundances and their radial behaviors are well reproduced.

This is not the whole story, obviously, since we do not consider the presence of a bulge and do not discuss the halo populations, in particular the globular clusters, important candles for the prehistory of galactic evolution. We are working on them.

We thank the referee for the accurate reading and sharp suggestions. This research has made use of the NASA/IPAC Extragalactic Database (NED), which is operated by the Jet Propulsion Laboratory, Caltech, under contract with the National Aeronautics and Space Administration. This work has been partially supported by Action Integrada EspañaItalia A-28, the Spanish DGICYT project PB 93-052, ASI, and MURST.

\section{APPENDIX}

\section{CHOICE OF RATE COEFFICIENTS AND UNIQUENESS}

The complexity of the system of equations reflects the need to incorporate an enormous variety of processes into any model for a galaxy. This is partly because there are so many individual constituent phases and partly because the system is not monolithic. The halo and disk (and thick disk and bulge) must be separately accounted for. In addition, there are many interconversion processes, each of which may be studied in detail at a local level, but all of which must act in concert to produce what we see as the long-term and large-scale development of a galaxy as a whole. It is true that the number of parameters is high, but such an objection is justified if the parameters are all freely set. There are severe constraints-both from stellar and interstellar evolution and from the studies that have been done on the individual processes-that prescribe a large number of the governing parameters. In Shore, Ferrini, \& Palla (1987), in the previous papers of this series, and in Shore \& Ferrini (1995), we outlined how some parameters are determined in advance of the model calculations and the others are determined post hoc from comparison with observations. We computed several models, for a number of extreme variations of the parameters; to state the result of this exploration at the outset, the system of equations turns out to be sensitive to comparatively few rates. The critical parameters are the rate of infall and the rate of star formation from molecular clouds.

For the present problem of radial abundance distributions, the system of equations has eight different parameters, related with the star formation rate and with the infall rate. However, five parameters are fixed from the solar neighborhood study (Papers I, II), thanks to the large number of data used as constraints for the model. Hence three virtually free parameters remain: the collapse time of the gas from the halo to the disk and the star formation and cloud formation efficiencies, $\epsilon_{H}$ and $\epsilon_{\mu}$. We fix the values for the collapse time in each galaxy from the solar value, taking into account the dynamical aspects of the problem. For the efficiencies $\epsilon_{H}$ and $\epsilon_{\mu}$, we use the Ferrini \& Galli (1988) analysis of the star formation rate for different Hubble types to restrict the possible values. Finally, we compute models for every galaxy by varying these two efficiencies around the values previously determined: the allowed variations of these efficiencies are small, otherwise some radial distribution of data would not be fitted.

The only way to justify another selection of parameters is to change the collapse time and efficiencies at the same time. If a shorter collapse time is selected, we need larger efficiencies in order to reach the level of observed elemental abundances and the observed gas consumption. But in this case a more massive and enriched halo than that observed is obtained.

Adler, D. S., \& Liszt, H. S. 1989, ApJ, 339, 836

Baiesi-Pillastrini, G. C. 1987, A\&A, 172, 375

Begeman, K. 1987, Ph.D. thesis, Univ. Groningen

Belley, J., \& Roy, J.-R. 1992, ApJS, 78, 61

Blair, W. P., Kirshner, R. P., \& Chevalier, R. A. 1981, ApJ, 247, 879

Bonnarel, F., Boulesteix, J., Georgelin, Y. P., Lecoarer, E., Marcelin, M., Bacon, R., \& Monnet, G. 1988, A\&A, 189,59

Bothun, G. D., \& Gregg, M. D. 1990, ApJ, 350,73

Braun, R. 1991, ApJ, 372, 54

Bronfman, L., Cohen, R. S., Alvarez, H., May, J., \& Thaddeus, P. 1988, ApJ, 324, 248

Caldwell, N., Kennicutt, R., Phillips, A. C., \& Schommer, R. A. 1991, ApJ, 370,526

Campos-Aguilar, A., Prieto, M., \& Garcia, C. 1993, A\&A, 276,16

Carignan, C., \& Freeman, K. C. 1985, AJ, 294, 494

Clarke, C. J. 1989, MNRAS, 238, 283

1991, MNRAS, 249,704

Clayton, D. D. 1987, ApJ, 315, 451

. 1988, MNRAS, 234, 1

\section{REFERENCES}

Corradi, R. L. M., Boulesteix, J., Bosma, A., Cappaccioli, M., Amran, P., \& Marcelin, M. 1991, A\&A, 244, 27

Corradi, R. L. M., \& Capaccioli, M. 1990, A\&A, 237, 36

DeGioia-Eastwood, K., Grasdalen, G. L., Strom, S. E., \& Strom, K. M. 1984, ApJ, 278, 564

Deharveng, L., Caplan, J., Lequeux, J., Azzopardi, M., Breysacher, J., Tarenghi, M., \& Westerlund, B. 1988, A\&AS, 73,407

Dennefeld, M., \& Kunth, D. 1981, AJ, 86, 989

Deul, E. R., \& den Hartog, R. H. 1990, A\&A, 229, 362

Devereux, N. A., \& Young, J. S. 1991, ApJ, 371, 515

Díaz, A. I. 1989, in Evolutionary Phenomena in Galaxies, ed. J. E. Beckman \& B. E. J. Pagel (Cambridge: Cambridge Univ. Press), 377

. 1994, in Violent Star Formation from 30 Doradus to QSOs, ed. G. Tenorio-Tagle (Cambridge: Cambridge Univ. Press), 105

Díaz, A. I., Terlevich, E., Vílchez, J. M., Pagel, B. E. J., \& Edmunds, M. G. 1991, MNRAS, 253, 245

Díaz, A. I., \& Tosi, M. 1984, MNRAS, 208, 365

Edmunds, M. G. 1990, MNRAS, 246, 678

Edmunds, M. G., \& Roy, J. R. 1993, MNRAS, 261, L17 
Edvardsson, B., Andersen, J., Gustafsson, B., Lambert, D. L., Nissen, P. E., \& Tomkin, J. 1993, A\&A, 275, 101

Elmegreen, D. M., \& Elmegreen, B. G. 1987, ApJ, 314, 3

Ferrini, F., \& Galli, D. 1988, A\&A, 195, 27

Ferrini, F., Matteucci, F., Pardi, C., \& Penco, U. 1992, ApJ, 387, 138 (Paper I)

Ferrini, F., Mollá, M., Pardi, M. C., \& Díaz, A. I. 1994, ApJ, 427, 745 (Paper III)

Ferrini, F., \& Poggianti, B. M. 1993, ApJ, 410, 44

Forbes, D. A., \& Whitmore, B. C. 1989, ApJ, 339, 657

Friedli, D., Benz, W., \& Kennicutt, R. 1994, ApJ, 430, L105

Gallagher, J. S., Hunter, D. A., \& Tutukov, A. V. 1984, ApJ, 284, 544

Götz, M., \& Köppen, J. 1992, A\&A, 262, 455

Güsten, R., \& Mezger, P. G. 1982, Vistas Astron., 26, 159

Huchtmeier, W. K., \& Richter, O.-G. 1989, A General Catalog of H I Observations of Galaxies: The Reference Catalog (New York: Springer)

Jones, B. J. T., \& Wyse, R. F. G. 1983, A\&A, 120,165

Kamphuis, J., \& Briggs, F. 1992, A\&A, 253, 335

Kennicutt, R. C., Jr. 1989, ApJ, 344, 685

Koper, E., Dame, T. M., Israel, F. P., \& Thaddeus, P. 1991, ApJ, 383, L11

Kwitter, K. B., \& Aller, L. H. 1981, MNRAS, 195,939

Lacey, C. G., \& Fall, S. M. 1983, MNRAS, 204,791 1985, MNRAS, 290, 154

Lada, C. L., Margulis, M., Sofue, Y., Nakai, N., \& Handa, T. 1988, ApJ, 328,143

Lambert, D. L. 1989, in AIP Conf. Proc. 183, Cosmic Abundances of Matter, ed. C. J. Waddington (New York: AIP), 168

Lequeux, J. 1983, A\&A, 125, 394

Martin, P., \& Roy, J. R. 1994, ApJ, 424, 599

Matteucci, F., \& François, P. 1989, MNRAS, 239, 885

McCall, M. L. 1982, Ph.D. thesis, Univ. Texas, Austin

McCall, M. L., Rybski, G. A., \& Shields, G. A. 1985, ApJS, 57, 1

McGaugh, S. S. 1994, ApJ, 426, 135

Mead, K. N., Kutner, M. L., \& Evans, N. J., II. 1990, ApJ, 354, 492

Meyer, J. P. 1989, in AIP Conf. Proc. 183, Cosmic Abundances of Matter, ed. C. J. Waddington (New York: AIP), 245

Minniti, D., Olszewski E. W., \& Rieke, M. 1994, in Third ESO/CTIO Workshop on the Local Group, ed. A. Layden \& J. Storm (Garching: ESO), in press

Mollá, M., \& Ferrini, F. 1995, ApJ, 454, 726 (Paper V)

Natali, G., Pedichini, F., \& Righini, M. 1992, A\&A, 256,79

Newton, K. 1980, MNRAS, 190, 689

Oey, M. S., \& Kennicutt, R. C., Jr. 1993, ApJ, 411, 137

Pagel, B. E. J. 1989a, in Evolutionary Phenomena in Galaxies, ed. J. E. Beckman \& B. E. J. Pagel (Cambridge: Cambridge Univ. Press), 201 1989 b, Rev. Mexicana Astron. Astrofis., 18,161

1991, in Nuclei in the Cosmos, ed. H. Oberhummer (Berlin: Springer), 89

Pagel, B. E. J., Edmunds, M. G., Blackwell, D. E., Chun, M. S., \& Smith, G. 1979, MNRAS, 189, 95

Pardi, C., \& Ferrini, F. 1994, ApJ, 421, 491 (Paper II)

Pardi, C., Ferrini, F., \& Matteucci, F. 1995, ApJ, 444, 207 (Paper IV)

Peimbert, M., \& Serrano, J. 1982, MNRAS, 198, 563

Persic, M., \& Salucci, P. 1991, ApJ, 368, 60
Phillips, S., \& Edmunds, M. G. 1991, MNRAS, 251, 84

Renzini, A., \& Voli, M. 1981, A\&A, 94, 175

Rogstad, D. H., Crutcher, R. M., \& Chu, K. 1979, ApJ, 229,509

Rumstay, K. S., \& Kaufman, M. 1983, ApJ, 274, 611

Ryden, B. S. 1988, ApJ, 329, 589

Ryden, B. S., \& Gunn, J. E. 1987, ApJ, 318, 15

Ryder, S. D. 1995, ApJ, 444, 610

Sage, L. J. 1993, A\&A, 272, 123

Salucci, P., \& Frenk, C. S. 1989, MNRAS, 237, 247

Sandage, A. 1986, A\&A, 161, 89

Sandage, A., \& Tammann, G. A. 1987, A Revised Shapley-Ames Catalog of Bright Galaxies, Carnegie Inst. Washington Publ. 635 (2d ed.; Washington: Carnegie Inst. Washington)

Sanders, D. B., Solomon, P. M., \& Scoville, N. Z. 1984, ApJ, 276, 182

Shore, S. N. 1983, ApJ, 265, 202

Shore, S. N., \& Ferrini, F. 1995, Fundam. Cosmic Phys., 16, 1

Shore, S. N., Ferrini, F., \& Palla, F. 1987, ApJ, 316, 663

Simien, F., \& de Vaucouleurs, G. 1986, ApJ, 302, 564

Sodroski, T. J. 1991, ApJ, 366, 95

Sommer-Larsen, J., \& Yoshii, Y. 1989, MNRAS, 238, 133

Tacconi, L. J., \& Young, J. S. 1986, ApJ, 308, 600 1989, ApJS, 71, 455

Thielemann, F. K., Nomoto, K., \& Hashimoto, H. 1994, in Supernovae: Les Houches 54, ed. S. A. Bludman, R. Mochkovitch, \& J. Zinn-Justin (Amsterdam: North-Holland), 629

Tinsley, B. M. 1980, Fundam. Cosmic Phys., 5, 287

Tosi, M., \& Díaz, A. I. 1985, MNRAS, 217, 571 1990, MNRAS, 246, 616

Tully, R. B. 1988, Nearby Galaxies Catalog (Cambridge: Cambridge Univ. Press)

Verter, F., \& Hodge, P. 1995, ApJ, 446, 616

Vila-Costas, B., \& Edmunds, M. G. 1992, MNRAS, 259, 121

Vílchez, J. M., \& Pagel, B. E. J. 1988, MNRAS, 231, 257

Vílchez, J. M., Pagel, B. E. J., Díaz, A. I., Terlevich, E., \& Edmunds, M. G. 1988, MNRAS, 235, 633

Wakker, B. P., \& Adler, D. S. 1995, AJ, 109, 134

Walterbos, R. A. M., \& Braun R. 1992, A\&AS, 92, 625

Wevers, B. M. H. R., van der Kruit, P. C., \& Allen, R. J. 1986, A\&AS, 66, 505

Wilson, C. D. 1995, ApJ, 448, L97

Wilson, C. D., \& Scoville, N. 1991, ApJ, 370, 184

Wilson, C. D., Scoville, N., Freedman, W. L., Madore, B. F., \& Sanders, D. B. 1988, ApJ, 333, 611

Wilson, C. D., Scoville, N., \& Rice, W. 1991, AJ, 101,1293

Wyse, R. F. G., \& Silk, J. 1989, ApJ, 339, 700

Young, J. S., \& Knezek, P. M. 1989, ApJ, 347, L55

Young, J. S., Schloerb, F. P., Kenney, J. D., \& Lord, S. D. 1986, ApJ, 304, 443

Young, J. S., \& Scoville, N. Z. 1982, ApJ, 260, L11

. 1991, ARA\&A, 29, 581

Young, J. S., Scoville, N. Z., \& Brady, E. 1985, ApJ, 288, 487

Zaritsky, D. 1992, ApJ, 390, L73

Zaritsky, D., Elston, R., \& Hill, J. M. 1989, AJ, 97,97

Zaritsky, D., Kennicutt, R. C., Jr., \& Huchra, J. P. 1994, ApJ, 420, 87 